\title{
Genomic insights on the contribution of balancing selection and local adaptation to the long-term survival of a widespread living fossil tree, Cercidiphyllum japonicum
}

\section{Shanshan Zhu ${ }^{1}$ (D), Jun Chen ${ }^{1}$ (D), Jing Zhao ${ }^{2} *$ (D), Hans Peter Comes ${ }^{3}$ (D), Pan Li ${ }^{1}$ (D), Chengxin Fu ${ }^{1}$, Xiao Xie $^{4}$ (D), Ruisen Lu ${ }^{1}$, Wuqin Xu ${ }^{1}$, Yu Feng ${ }^{1}$, Wenqing Ye ${ }^{1}$, Shota Sakaguchi ${ }^{5}$ (D), Yuji Isagi ${ }^{6}$ (D), Linfeng Li ${ }^{7}$ (D), Martin Lascoux ${ }^{8}$ (D) and Yingxiong Qiu ${ }^{1}$}

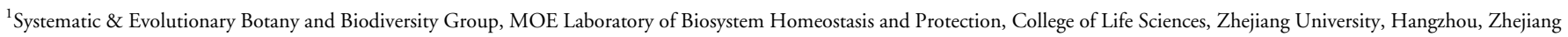

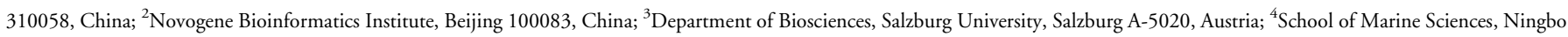
University, Ningbo, Zhejiang 315211, China; ${ }^{5}$ Graduate School of Human and Environmental Studies, Kyoto University, Yoshida-nihonmatsu-cho, Sakyo-ku, Kyoto 606-8501, Japan; ${ }^{6}$ Graduate School of Agriculture, Kyoto University, Kitashirakawa-Oiwake-cho, Sakyo-ku, Kyoto 606-8501, Japan; ${ }^{7}$ Ministry of Education Key Laboratory for Biodiversity Science and Ecological Engineering, School of Life Sciences, Fudan University, Shanghai 200433, China; ${ }^{8}$ Plant Ecology and Evolution, Department of Ecology and Genetics and Science for Life Laboratory, Uppsala University, Norbyvägen 18D, Uppsala 75236, Sweden

Author for correspondence: Yingxiong Qiu Tel: +8657188981703 Email: qyxhero@zju.edu.cn

Received: 14 January 2020 Accepted: 27 June 2020

New Phytologist (2020) 228: 1674-1689 doi: $10.1111 / n p h .16798$

Key words: adaptation, balancing selection, demographic history, living fossil, selective sweeps, whole-genome sequencing.

\section{Summary}

- 'Living fossils' are testimonies of long-term sustained ecological success, but how demographic history and natural selection contributed to their survival, resilience, and persistence in the face of Quaternary climate fluctuations remains unclear.

- To better understand the interplay between demographic history and selection in shaping genomic diversity and evolution of such organisms, we assembled the whole genome of Cercidiphyllum japonicum, a widespread East Asian Tertiary relict tree, and resequenced 99 individuals of $C$. japonicum and its sister species, Cercidiphyllum magnificum (Central Japan). - We dated this speciation event to the mid-Miocene, and the intraspecific lineage divergence of C. japonicum (China vs Japan) to the Early Pliocene. Throughout climatic upheavals of the late Tertiary/Quaternary, population bottlenecks greatly reduced the genetic diversity of $C$. japonicum. However, this polymorphism loss was likely counteracted by, first, long-term balancing selection at multiple chromosomal and heterozygous gene regions, potentially reflecting overdominance, and, second, selective sweeps at stress response and growth-related genes likely involved in local adaptation.

- Our findings contribute to a better understanding of how living fossils have survived climatic upheaval and maintained an extensive geographic range; that is, both types of selection could be major factors contributing to the species' survival, resilience, and persistence.

\section{Introduction}

Some species, for instance, coelacanths, horseshoe crabs, maidenhair tree (Ginkgo biloba), or dawn red wood (Metasequoia), survived for millions of years with little morphological change. For those 'anomalous forms that endured to the present day', Darwin (1859) coined the term 'living fossils'. Living fossils are not easy to define unambiguously, but, apart from their morphological stasis and persistence over long periods of evolutionary time, they also tend to have distribution ranges that are currently more restricted than in the geologic past (Werth \& Shear, 2014; Lidgard \& Love, 2018). As already foreseen by Darwin, such organisms provide excellent materials to address questions on

*These authors contributed equally to this work. species evolution. In particular, they can offer insights into a wide array of adjustments (e.g. morphological, physiological, genetic, demographic) that may have been instrumental in adaptation and survival through numerous episodes of environmental change (Werth \& Shear, 2014). One can ask, for instance, whether and how living fossils responded to the climatic oscillations of the Quaternary (Grandcolas et al., 2014). Did their effective population sizes contract and expand, as they did in many species, or did they remain rather stable through these periods of climatic upheaval, as reported for others (Kryvokhyzha et al., 2019)? Furthermore, it would be interesting to know how much of their genome is directly or indirectly influenced by natural selection, or, more specifically, which selective forces are shaping genomic variation and driving local adaptation (Kern \& Hahn, 2018). 
The greatest concentration of 'living fossil' plants presently occurs in the warm-temperate forests of East Asia (Axelrod, 1998; Manchester et al., 2009). Here, most of these Tertiary relict trees (e.g. G. biloba, Davidia involucrata, Emmenopterys henryi, Euptelea pleiosperma, Liriodendron chinense, Tetracentron sinense) are confined to relatively narrow, discontinuous ranges in subtropical China, where they often occur in small, isolated mountain populations on unstable sites, such as scree slopes and eroded rocky stream banks (reviewed in Shang et al., 2016). Earlier phylogeographic studies, primarily based on organellar markers, indicated that these Tertiary relict trees, indeed, experienced repeated range contractions and expansions associated with Quaternary glacial-interglacial cycles (Qiu et al., 2011; Sun et al., 2014; Ma et al., 2015; Cao et al., 2016; Zhang et al., 2016; Shen et al., 2019). However, with the exception of a couple of studies (Tsumura et al., 2012; Zhang et al., 2016; Zhao et al., 2019), there have so far been limited attempts to link those demographic changes with adaptive responses, even though one could expect that both are associated (Shaw \& Etterson, 2012). As shown by a recent study in G. biloba (Zhao et al., 2019), the advance of whole-genome sequencing technologies has dramatically increased our ability to understand the joint effects of demography and selection on the genetic polymorphisms of a Tertiary relict tree. By taking advantage of Ginkgo's reference genome, these authors inferred multiple population declines but also recoveries of the species in China over late Tertiary/Quaternary timescales. Moreover, signatures of positive selection were detected at genes involved in abiotic (e.g. climate) and biotic (e.g. pathogen defence) stresses (Zhao et al., 2019). Together, these results suggest that both demographic history and natural selection contributed to the species' long-term persistence and resilience. However, it would be premature to generalize from a single study, considering the great variability of evolutionary responses to Quaternary environmental change across species (e.g. Petit et al., 2003). Hence, further population genomics work is required to elucidate the interplay between historical demographic and selective factors in shaping the genetic diversity and evolution of East Asia's Tertiary relict flora.

To fill this gap, we report here the genome draft and population genomic structure of Cercidiphyllum japonicum Sieb. et Zucc., which, much in contrast to G. biloba, is the epitome of a widespread 'living fossil' tree in East Asia (Qi et al., 2012). This diploid $(2 n=38)$, dioecious, and wind-pollinated/dispersed tree is widely distributed across subtropical (Central/ South/East) China all the way to north Japan (Hokkaido), and thus covers various climate and vegetation zones, including warm-temperate deciduous, cool-temperate, and subalpine forests (Fu \& Endress, 2001; Isagi et al., 2005; Krassilov, 2010). There is phylogeographic evidence to suggest that C. japonicum has survived in East Asia for at least $5 \mathrm{Myr}$, and experienced dramatic population fluctuations in both China and Japan during the Quaternary glacial-interglacial cycles (Qi et al., 2012). Based on population genetics theory (Hedrick, 2006) and empirical data (Bernatchez, 2016; GonzálezMartínez et al., 2017; Lin et al., 2017; Gossmann et al., 2019), one might, therefore, expect these climate-induced fluctuations in population size and migration rates to have been accompanied by bouts of natural selection.

In this study, we generated a chromosome-level de novo assembly of the whole genome of C.japonicum and resequenced 82 individuals, representing 24 sampled populations (localities) from China (eight) and Japan (16). For comparison, we also resequenced 17 individuals (four populations) of C. magnificum Nakai, which is the only other species of the genus and is today restricted to the cool-temperate/subalpine forests of Central Honshu/Japan (Qi et al., 2012). Hence, we first inferred the demographic history of the two species and then tested for the presence of genome-wide selection in the Chinese vs Japanese lineages of C. japonicum. Overall, our study provides evidence that this exceptionally widespread 'living fossil' repeatedly recovered from demographic bottlenecks over late Tertiary/Quaternary periods of adverse climate conditions, and that its survival might have been facilitated through the maintenance of genetic diversity by genome-wide balancing selection and selective sweeps at particular genes involved in local adaptation.

\section{Materials and Methods}

\section{Genome sequencing, assembly, and annotation}

For whole-genome sequencing, we collected fresh young leaves of an adult C.japonicum tree from Tianmu Mountain, Zhejiang, China $\left(30.326^{\circ} \mathrm{N}, 119.442^{\circ} \mathrm{E}\right)$. We sequenced and assembled the genome using a combination of PacBio single-molecule long reads and Illumina short reads and further improved the assembly by adding $10 \times$ Genomics Linked-Reads. Then, a Hi-C library was sequenced to assist the chromosome-level assembly. The completeness of the genome assembly was assessed using both Cegma (Parra et al., 2007) and Busco (Simão et al., 2015). Gene prediction was conducted through a combination of homologybased inference, ab initio prediction, and transcripts from RNA sequencing (RNA-Seq). We also used a combination of homology-based and de novo approaches to identify transposable elements (TEs) in the C.japonicum genome. For details, see Supporting Information Methods S1 and Tables S1-S10.

\section{Genomic synteny and gene family analyses}

MCSCANX (Wang et al., 2012) was employed to identify syntenic blocks within and between the genomes of C.japonicum, Vitis vinifera and Kalanchoe fedtschenkoi. We also calculated the fourfold synonymous third-codon transversion (4DTv) rate for orthologous/paralogous genes in syntenic blocks to identify putative whole genome duplication events in C.japonicum (Maere et al., 2005). OrthoMCL v.2.0 ( $\mathrm{Li}$ et al., 2003) was used to identify paralogues and orthologues between C.japonicum and 17 other sequenced angiosperm species (see Table S11). The 413 single-copy orthologous genes of all 18 species (Fig. S1) were used to reconstruct a maximum likelihood (ML) phylogeny with RAxML v.8.0 (Stamatakis, 2014). Gene family expansion and contraction analysis was performed with CAFÉ v.3.1 (Han et al., 2013). 


\section{Divergence time and mutation rate estimation}

We derived 287 single-copy orthologous genes among 19 angiosperm species, including C.japonicum and C. magnificum (from transcriptome data), for phylogenetic analysis and molecular dating. For each species, the genes were concatenated into a 'supergene' and aligned using MusCle v.3.8.31 (Edgar, 2004). The alignment was curated with GBLOCKs v.1.0.4 (Castresana, 2000) and then used to perform phylogenetic analysis using RAxML v.8.0 with 1000 bootstrap replicates. We further estimated the divergence time for each ancestral node of these 19 species in BEAST v.1.8.1 (Drummond et al., 2012), using four calibration times. The mutation rate was estimated as $\mu=D / 2 \times T$, where $D$ is the observed frequency of pairwise differences between C. japonicum and C. magnificum, and $T$ is their estimated divergence time (for details, see Methods S1).

\section{Resequencing, mapping, and genotyping}

For whole-genome resequencing, individual leaf samples were collected from a total of 24 populations $(n=82)$ of $C$. japonicum throughout its range in China ( $n=31$; eight populations) and Japan ( $n=51 ; 16$ populations) and four populations $(n=17)$ of C. magnificum from its narrow range in Central Honshu/Japan (Fig. S2). Raw sequence reads of each of those 99 individuals were mapped to the reference genome of $C$. japonicum using BWA v.0.7.7 (Li \& Durbin, 2010). SAMTools v.0.1.19 (Li \& Durbin, 2009) was used for sorting and removing PCR duplicates. Raw single nucleotide polymorphisms (SNPs) were called with SAMтооLs, using the command 'samtools mpileup q 1 C 50 S D m 2 F 0.002 u'. Three filtering steps were performed to reduce false positives from SNP and genotype calling: (1) removal of SNPs with quality score of $<20$; (2) removal of SNPs with more than two alleles; and (3) assignment of individual genotypes with reads less than one-third of mean depth as 'missing'. We also applied the following criteria to obtain high-quality genotype calls. Only SNPs containing $\leq 20 \%$ of missing data (missing ratio) and minor allele frequencies $(\mathrm{MAF}) \geq 0.01$ were retained for population genetic analyses in the combined data set of C. japonicum $(n=82)$ and C. magnificum $(n=17)$. To evaluate whether our inference of population structure is robust to the calling of rare variants due to unequal sample sizes of the two species, we also applied the aforementioned filtering criteria with the exception of MAF $\geq 0.05$ to each species. We performed population structure analysis using both data sets.

\section{Genetic relationship and population structure analyses}

An approximately ML phylogenetic tree was constructed based on the total SNP data set of all 99 accessions of C. japonicum/ C. magnificum using FastTree v.2 (Price et al., 2010). Population structure was further assessed by Bayesian clustering in Admixture v.1.23 (Alexander et al., 2009). The number of clusters $K$ was set to vary from 2 to 6 , and the best value was chosen based on the lowest cross-validation error. We also assembled complete chloroplast genome sequences from all whole-genome re-sequenced Cercidiphyllum individuals $(n=99)$, following procedures described in Huang et al. (2014) (for details see Methods S2). We also conducted phylogenetic analysis based on these 99 chloroplast genome sequences with four outgroups (Bergenia scopulosa, Heuchera parviflora var. saurensis, Sedum oryzifolium and Sedum sarmentosum) using RAxML. Considering coding sequence regions only, we identified a total of 30 chloroplast haplotypes for this dataset; genealogical relationships among these 'chlorotypes' were assessed through a 95\% statistical parsimony network, using the program TCS, as implemented in POPART v.1.7 (Leigh \& Bryant, 2015; for details, see Methods S2).

\section{Reconstruction of demographic history}

We used the pairwise sequentially Markovian coalescent (PSMC) method (Li \& Durbin, 2011) to infer historical changes in effective population size $N_{\mathrm{e}}$ based on the reference genome of C. japonicum and 28 resequenced individuals from each locality of C. japonicum $(n=24) / C$. magnificum $(n=4)$. We also used the sequentially Markovian method (SMC++; Terhorst et al., 2017), which uses polymorphism data of multiple unphased genomes, to reconstruct the population size histories of Chinese and Japanese C. japonicum as well as C. magnificum. SMC++ is more powerful than PSMC in estimating changes of $N_{\mathrm{e}}$ over shallow timescales. Finally, we implemented coalescent simulations of six divergence models in FASTSIMCOAL2 (Excoffier et al., 2013) to infer the past demographic histories of the two Cercidiphyllum species using the joint site frequency spectrum (SFS; Fig. S3; for details see Methods S2).

\section{Genome-wide scanning for selection in C. japonicum}

To identify genomic regions potentially under selection in C. japonicum, we calculated average values of $\pi$, Tajima's $D$, $F_{\mathrm{ST}}$, and $D_{x y}$ across all populations per region (China vs Japan) using a genome-wide sliding window strategy $(100 \mathrm{~kb}$ in length with steps of $10 \mathrm{~kb}$ ) as implemented in VCFTOOLS v.0.1.15 (Danecek et al., 2011). Windows with $z$-score-normalized $F_{\mathrm{ST}}>2(95 \%$ tail) were considered to be candidate regions likely driven by diversifying selection for local adaptation. Then we merged the neighboring windows into regions and filtered those regions with a cutoff of length $>1 \mathrm{Mb}$ and mean Tajima's $D<-0.95$ ( $10 \%$ tail). The mean of Tajima's $D$ was calculated from all windows with more than 100 SNPs within the merged regions of the samples by averaging their $D$ values. To identify candidate regions under selective sweeps, we first performed haplotype phasing in MACH v.1.0 (Li et al., 2010) with parameters 'states 100' and 'rounds 50'. Linkage disequilibrium between pairs of SNPs within $1 \mathrm{Mb}$ was calculated by EMERALD (Quick et al., 2018). The likelihood ratio test statistic $T$ (Harris \& DeGiorgio, 2019) was used to test for selective sweeps based on haplotype diversity decrease at surrounding sites. Putative selective sweep regions were selected based on $T>51.9$ (99.5\% tail).

We identified putative regions under balancing selection based on the distributions of Tajima's $D(95 \%$ tail, $>1.3$ and $>1.56$ 
for Chinese and Japanese populations, respectively), $\pi$ (95\% tail, $>0.0024$ and $>0.0023$, respectively), and $F_{\mathrm{ST}}$ ( $5 \%$ tail, $z$-score $<-0.92)$. Percentages of heterozygous sites were calculated for the four regions and the whole genome of each individual (see the Results section). Individuals were considered carriers of balancing polymorphism only if the percentage of heterozygous sites was $>30 \%$. This threshold was determined as the values of other individuals dropped greatly from $30 \%$ to below $20 \%$. We further validated signatures of long-term balancing selection using normalized $\beta$ statistics, as calculated in BETASCAN (https://github.c om/ksiewert/BetaScan). As shown by simulations (Siewert \& Voight, 2017), this measure provides greater power than other summary statistics for balancing selection, especially in nonequilibrium populations.

\section{Estimates of selective efficacy and distribution of fitness effects}

For each protein-coding gene, we also calculated the nucleotide diversity $\pi$ at nonsynonymous (zero-fold) and synonymous (four-fold) sites (i.e. $\pi_{\mathrm{N}}$ and $\pi_{\mathrm{S}}$, respectively) for the Japanese and Chinese populations of C.japonicum. The ratio of the average $\pi_{\mathrm{N}} / \pi_{\mathrm{S}}$ was then used as a measure of selection efficacy (James et al., 2017). It was also used in connection with the analysis of the candidate region of selective sweeps, since $\pi_{S}$ is affected by hitchhiking, with a stronger effect of hitchhiking in areas of low diversity (Castellano et al., 2018). The distribution of fitness effects (DFE) of new mutations (Eyre-Walker \& Keightley, 2007) was estimated by comparing the unfolded SFS between nonsynonymous and synonymous sites, using POLYDFE v.2 (Tataru \& Bataillon, 2018). Likelihoods of fitted DFE parameters, either constrained to be shared (invariant) across data sets or fitted independently for each data set, were then compared to test for significant differences in selection between the Chinese and Japanese populations. For each group, we first tested a model with deleterious mutations following a gamma distribution and then a full DFE model with both deleterious mutations still distributed according to a gamma distribution and beneficial mutations distributed according to an exponential distribution. Parameter estimates for the proportion of beneficial mutations $p_{\mathrm{b}}$, mean positive selective strength $S_{\mathrm{b}}$, shape of the gamma distribution $\beta$, and mean negative selective strength $S_{\mathrm{d}}$ were taken by averaging across values of both DFE models as weighted by the model's Akaike information criterion value (Posada \& Buckley, 2004).

\section{Genotype-environmental association analyses}

For the environmental data, we downloaded 19 bioclimatic variables for the period 1960-1990 from WorldClim v.1.4 (Hijmans et al., 2005) for each location of the Chinese and Japanese populations of C.japonicum. We performed principal component (PC) analysis (PCA) in R (R Development Core Team, 2018) to remove redundancies among correlated variables and keep most of the variance. The first six PCs (explaining $>99 \%$ of the total variance of the sampled populations) were then chosen to test for the correlation between environmental factors and SNP frequencies (MAF $\geq 0.05$; missing ratio $\leq 0.2$ ) using a latent factor mixed model (LFMM) implemented in the $\mathrm{R}$ package LFMM (Caye et al., 2019; see details in Methods S2). SNPs with correlated $P_{-}$ values (false discovery rate, FDR) $<0.05$ and effect scores $>10$ were regarded as significantly associated with environmental differentiation between China and Japan. In addition, we selected candidate genes harboring environmentally associated SNPs for Gene Ontology (GO) enrichment analysis using Arabidopsis thaliana orthologues at agriGO (Du et al., 2010). To test the significance of GO terms, we applied a Bonferroni-adjusted $P$-value $<0.05$ as the threshold.

\section{Results}

\section{Genome assembly and genome evolution of $C$. japonicum}

In total, $299 \mathrm{~Gb}$ data $(c .400 \times)$ were assembled into a high-quality genome of $719 \mathrm{Mb}$ for C. japonicum, with N50 of $494.88 \mathrm{~kb}$ (Tables S1-S3). About $90.18 \%(648 \mathrm{Mb})$ of the assembly was anchored to 19 pseudo-chromosomes (Figs 1, S4, S5). A total of 27393 gene models were predicted, and 26176 were verified by transcriptome sequences and existing homologous sequences (Tables S4-S10; Methods S1). Syntenic analysis revealed that C. japonicum experienced a whole-genome triplication event, referred to as gamma $(\gamma)$ in core eudicots (Jaillon et al., 2007; Figs 2a,b, S6; Methods S1). A total of 72 gene families in C. japonicum had undergone significant expansions $(P<0.05$; Fig. S7). These gene families were mostly enriched with 'responding to stimulus' and 'nucleotide binding' functional subcategories, and associated with disease resistance genes (e.g. $R$-genes) encoding nucleotide-binding site-leucine-rich repeat proteins (Tables S11-S13). The ML tree inferred from 287 single-copy orthologues among 19 angiosperm species confirmed the monophyly of Cercidiphyllum as sister to a clade comprising the two other members of Saxifragales; that is, K. fedtschenkoi and Rhodiola crenulata. The crown age of Cercidiphyllum was estimated at 13.41 million years ago (Ma; $95 \%$ highest posterior density (HPD): 15.02-11.77 Ma; Fig. 2c). The estimated 4DTv rate of the Cercidiphyllum sequences was c. $5.35 \times 10^{-10}$ per site per year (range: $4.77 \times 10^{-10}-6.09 \times 10^{-10}$ ), which is lower than that of other trees (e.g. $1.16 \times 10^{-9}$ for Populus trichocarpa (Tuskan et al., 2006); $1.09 \times 10^{-9}$ for Salix suchowensis (Dai et al., 2014); and $2.50 \times 10^{-9}$ for Juglans species (Bai et al., 2018)).

\section{Population structure and admixture}

Whole-genome resequencing resulted in a total of 10148438142 paired-end reads for C. japonicum $(n=82)$ and 2127448854 for C. magnificum $(n=17)$. About $89 \%$ of C. japonicum sequence reads and $83 \%$ of $C$. magnificum sequence reads were accurately mapped to the reference genome. The mean sequencing depth was $22 \times$ and $24 \times$ in samples of C. japonicum and C. magnificum, respectively (Table S14). After filtering, we detected a total of 6923574 SNPs across the 99 individuals 


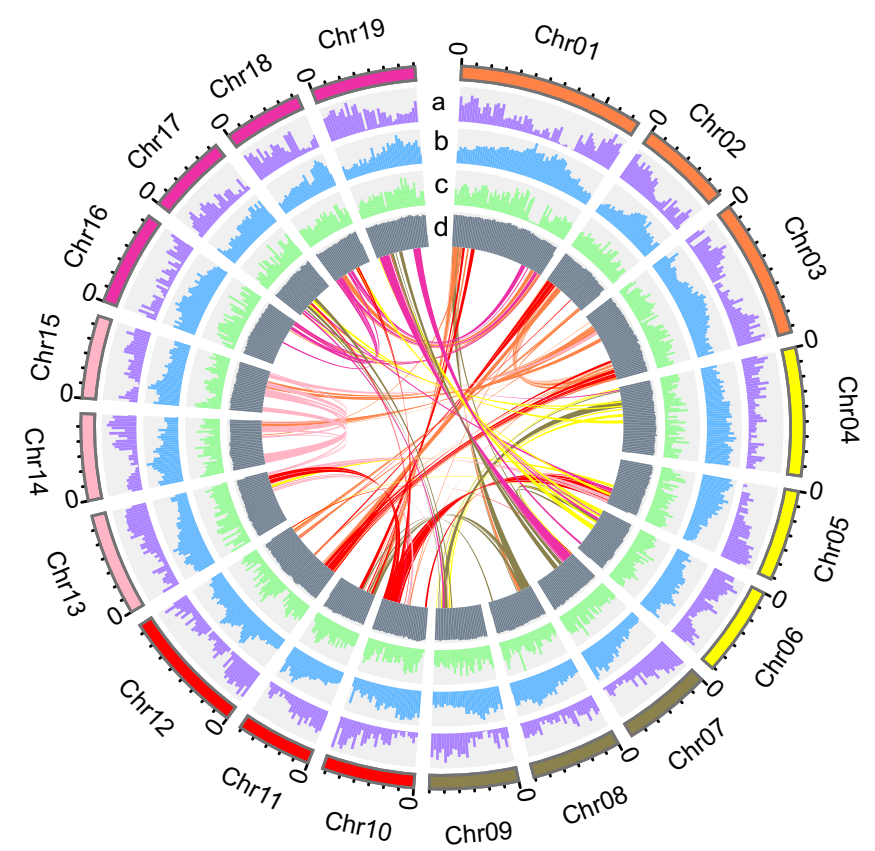

Fig. 1 Circos plot of chromosome-level assembly of the Cercidiphyllum japonicum genome. The circles, from outermost to innermost, show (a) gene density, (b) repeat element density, (c) single nucleotide polymorphism density for population genomic analysis, and (d) GC content. The four metrics are calculated in $1 \mathrm{Mb}$ sliding windows. The central lines show the syntenic regions within the $C$. japonicum genome.

analyzed (Table S15). Not unexpectedly, among the 1080097 rare variants (i.e. SNPs found in only one individual and as homozygotes, or in two individuals and as heterozygotes), the majority $(957859 ; 88.7 \%)$ were present as heterozygotes. When filtering SNPs for each species with $\mathrm{MAF} \geq 0.05$ and missing ratio $\leq 0.2$ separately, we identified 2251212 and 590523 SNPs across the 82 C.japonicum samples and 17 C.magnificum samples, respectively.

The ML tree based on the aforementioned two data sets consistently recovered two highly supported main clusters corresponding strictly to species taxonomy (Figs 3a, S8; Tables S14, S15). Within C.japonicum, most Chinese accessions formed a separate cluster, except for those of the most eastern population (CTT). The corresponding ADMIXTURE analyses at $K=3-5$ (with $K=4$ being the optimal cluster number; see Fig. 3 b) revealed that CTT populations were genetically admixed with conspecific populations from south Japan (JSY, JSD, JST, JSE). At $K=5$, samples of Japanese C.japonicum were further subdivided into regional subclusters from south and north Japan, with central populations showing genetic admixture to some extent (Figs 3c, S8). By contrast, the ML phylogeny based on whole chloroplast genome sequences of all 99 accessions recovered two major clades: one composed of C.japonicum from China and south Japan, and the other containing C. japonicum from central-north Japan and C. magnificum (Fig. 3d). Similarly, a parsimony network of the 30 chloroplast haplotypes indicated a closer relationship of central-north Japanese C.japonicum to C. magnificum than to other conspecifics (Fig. 3e).

\section{Reconstructing past population dynamics}

Based on the PSMC analysis of the high-coverage reference genome of C. japonicum, the species' ancestral population started to expand from $c .10 \mathrm{Ma}$ until 3-2 Ma, followed by a severe bottleneck that lasted until c. $0.5 \mathrm{Ma}$, during which $N_{\mathrm{e}}$ was reduced by c. $65 \%$ (Fig. S9). Notably, C. magnificum followed a similar demographic trajectory of population growth and subsequent decline over the same periods, except that the bottleneck lasted 0.5 Myr longer and was even more severe (Fig. 4a). Based on the SMC++ analysis of all 99 whole-genome resequenced individuals, population sizes of each species increased rapidly after $c$. $20000 \mathrm{yr}$ вP and then started to decline c. 3000-2000 yr вP (Fig. S10).

According to the best-supported model (Figs 4b, S11; Tables S16, S17), C. japonicum and C. magnificum diverged from their common ancestor at the mid-Miocene, c. $10.33 \mathrm{Ma}(95 \%$ HPD: 10.96-6.73 Ma). The divergence of Chinese and Japanese C. japonicum occurred at the Early Pliocene, c. $4.17 \mathrm{Ma}(95 \%$ HPD: 4.88-3.93 Ma). Estimates of bidirectional gene flow revealed low levels of ancient gene flow between the common ancestor of Chinese/Japanese lineages of C.japonicum and C. magnificum (0.036/0.003; Fig. 4b). By contrast, after the divergence of Chinese and Japanese C.japonicum, estimates of gene flow between the two species were extremely low $\left(9.02 \times 10^{-6} / 7.53 \times 10^{-6}\right)$, and the same was true for the latter two lineages $\left(1.55 \times 10^{-5} / 1.29 \times 10^{-5}\right.$; Fig. 4b). According to this best-fit FASTSIMCOAL2 model, all three lineages experienced a bottleneck event during the Early to Middle Pleistocene (c. 1.6$1.1 \mathrm{Ma}$ ), resulting in estimates of current $N_{\mathrm{e}}$ that were only slightly smaller in the narrow endemic $C$. magnificum relative to Japanese and, to a lesser extent, Chinese C. japonicum (Fig. 4b).

\section{Selective sweeps increased genetic differentiation between Japanese and Chinese $C$. japonicum}

Across their entire genomes, Chinese and Japanese populations of C. japonicum exhibited similar levels of nucleotide diversity (mean $\pi=1.1 \times 10^{-3}$ and $9.9 \times 10^{-4}$, respectively), and low levels of inter-lineage differentiation $\left(F_{\mathrm{ST}}=0.07\right.$, compared with 0.72 between $C$.japonicum and C. magnificum; Fig. S12). Average linkage disequilibrium was low (mean $r^{2}$ within $1 \mathrm{~kb}$ was 0.18 and 0.19 for Chinese and Japanese populations, respectively) and decreased by half within $c .2 .5 \mathrm{~kb}$ (Fig. S13). When these two regional groups were subjected to an $F_{\mathrm{ST}}$-based genome-wide sliding window scan $(100 \mathrm{~kb}$ windows with steps of $10 \mathrm{~kb}), 2854$ outlier regions were identified with $z$-score-normalized $F_{\mathrm{ST}}$ values $>2$ (Fig. 5a). Of those potential selection regions, $19.7 \%$ (563) were located on Chr14, covering c. $19.3 \%$ of its total length, and thus significantly more than on other chromosomes $\left(4.3 \%\right.$ on average). Specifically, $86.5 \%$ of high $F_{\mathrm{ST}}$ windows were located in a region $(5-14 \mathrm{Mb})$ in which Japanese populations had significantly lower diversity than Chinese ones (mean $\pi=0.0008$ vs $0.0012, P<0.01$ ), as well as significantly lower negative values of Tajima's $D$ (mean: -0.23 vs 0.21 , $P<0.01)$. Notably, those differences were even more pronounced 
(a)

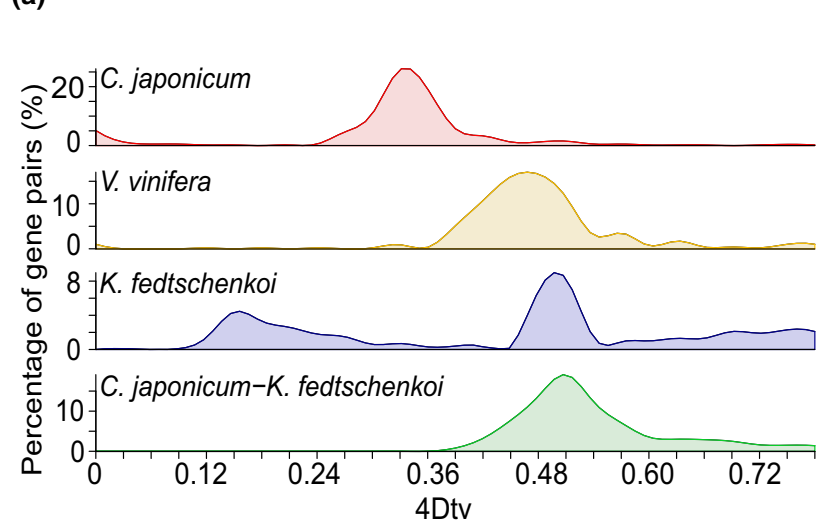

(c)

(c)

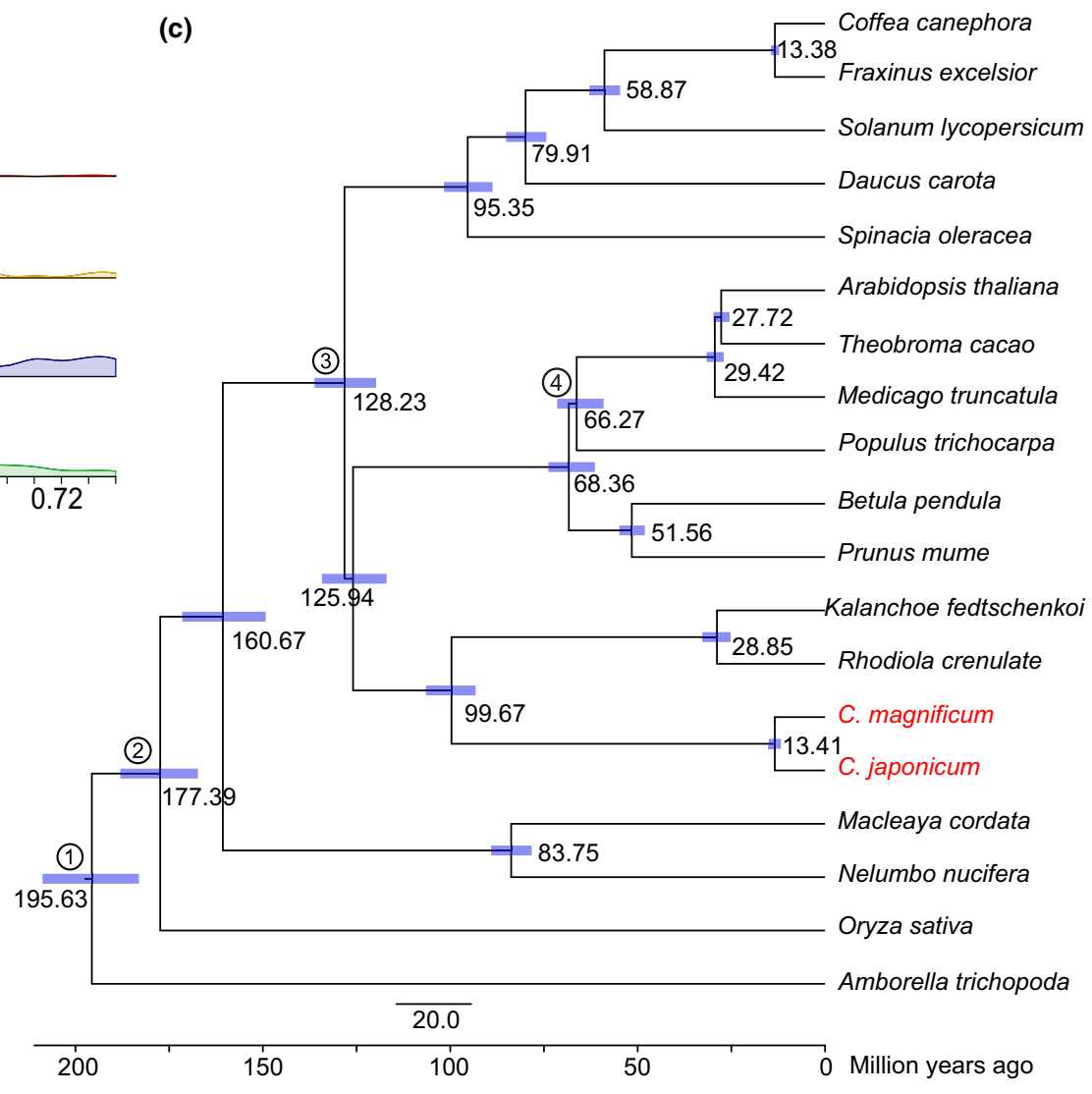

(b)

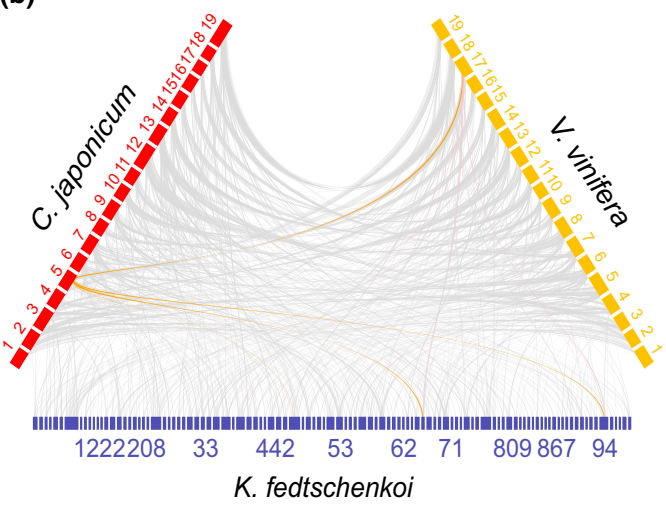

Fig. 2 Evolution of the Cercidiphyllum japonicum genome and gene families. (a) Distribution of the four-fold synonymous third-codon transversion rate (4DTv) for paralogous genes within the genomes of C. japonicum, Vitis vinifera, and Kalanchoe fedtschenkoi and orthologous genes between those of C. japonicum and $K$. fedtschenkoi. (b) Macrosynteny patterns showing that a typical ancestral region in C. japonicum can be tracked to up to one region in V. vinifera and to up to four regions in K. fedtschenkoi. (c) BEAST-derived chronogram of 19 angiosperm species, including C. japonicum and Cercidiphyllum magnificum, based on 287 single-copy orthologous genes. The number at each node represents the median divergence time (in million years ago, Ma), with blue bars representing the $95 \%$ highest posterior density interval. Numbers with circles represent calibration times of divergence between (1) Amborella trichopoda and Oryza sativa (168-194 Ma), (2) O. sativa and Populus trichocarpa (148-173 Ma), (3) Coffea canephora and Theobroma cacao (110-124 Ma), and (4) Arabidopsis thaliana and P. trichocarpa (97-109 Ma). Calibration times were obtained from the Timetree database (http://www.timetree.org/).

for the central region of Chr14 at $8.5-12 \mathrm{Mb}$ (mean $\pi$ : 0.0007 vs $0.0015, P<0.01$; Tajima's $D$ : -0.97 vs $-0.26, P<0.01$ ) (see Fig. 5b). This region also had the highest estimate of Nei's genetic distance $\left(D_{x y}=0.0037\right.$ vs $5.29 \times 10^{-4}$ for the whole genome) and contained all 189 fixed differences $D_{\mathrm{f}}$ between the Chinese and Japanese populations.

Taken together, these results suggest that selective sweeps took place in the Japanese populations but were absent or less pronounced in those from China. To ascertain these results, we performed two additional analyses. First, based on the $T$ statistic, we identified a mixture of hard sweeps (number of sweeping haplotypes $m=1)$ and soft sweeps $(m=3-7$; Fig. $5 b)$ in the region $8.5-12 \mathrm{Mb}$ of $\mathrm{Chr} 14$ in Japanese populations. Second, since hitchhiking effects can elevate $\pi_{\mathrm{N}} / \pi_{\mathrm{S}}$ in areas of low diversity (Castellano et al., 2018), we compared $\pi_{\mathrm{N}} / \pi_{S}$ ratios for each gene and each regional lineage separately. On average, values of $\pi_{\mathrm{N}} / \pi_{\mathrm{S}}$ across all chromosomes were rather low and similar (Japan: 0.29; China: 0.28). However, in the selective sweep region of Chr14 (8.5-12 Mb, including 41 genes; see details in
Table S18), this ratio was significantly larger for the Japanese than the Chinese populations (0.58 vs 0.48 ). Estimates of DFE further confirmed a small but still higher proportion of beneficial mutations $p_{\mathrm{b}}$ in Japan than in China $\left(2.8 \times 10^{-4}\right.$ vs $\left.2.4 \times 10^{-5}\right)$. On Chr14, this difference was even two orders of magnitude higher $\left(0.013\right.$ vs $\left.1.3 \times 10^{-4}\right)$. Moreover, mean positive selective strength $S_{\mathrm{b}}$ on Chr14 was higher in Japan $(0.076$ vs 0.019$)$. Such selective sweeps also elevated $F_{\mathrm{ST}}$ between the Japanese populations of C.japonicum and C. magnificum (see Fig. 5b).

A patch-like pattern of heterozygous sites across the $3.5 \mathrm{Mb}$ region of Chr14 (8.5-12 Mb) were detected in Japanese and Chinese C. japonicum (Fig. S14). For example, a majority of Japanese C. japonicum individuals shared a long stretch of homozygous alleles (type I) across the 'selective sweep' region of Chr14 (8.5$12 \mathrm{Mb}$; see an example of gene VLN4 in Fig. 5c). By contrast, about half of the Chinese individuals shared a different set of homozygous alleles (type II) across this region, whereas the rest appeared to be heterozygous for both allele types at various sites 
(a)

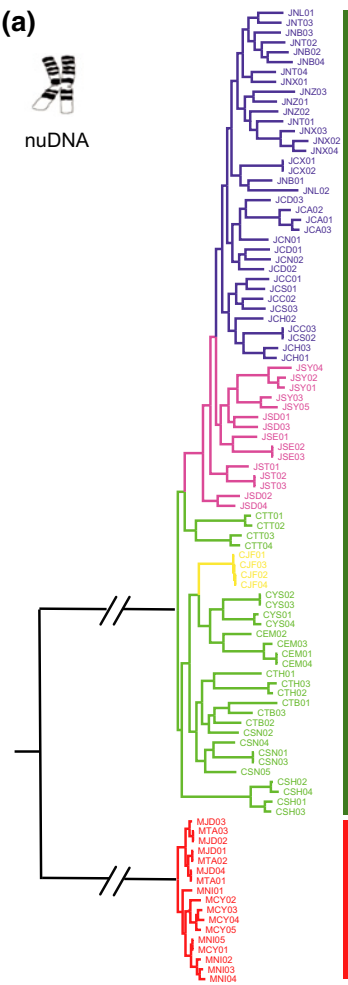

(d)

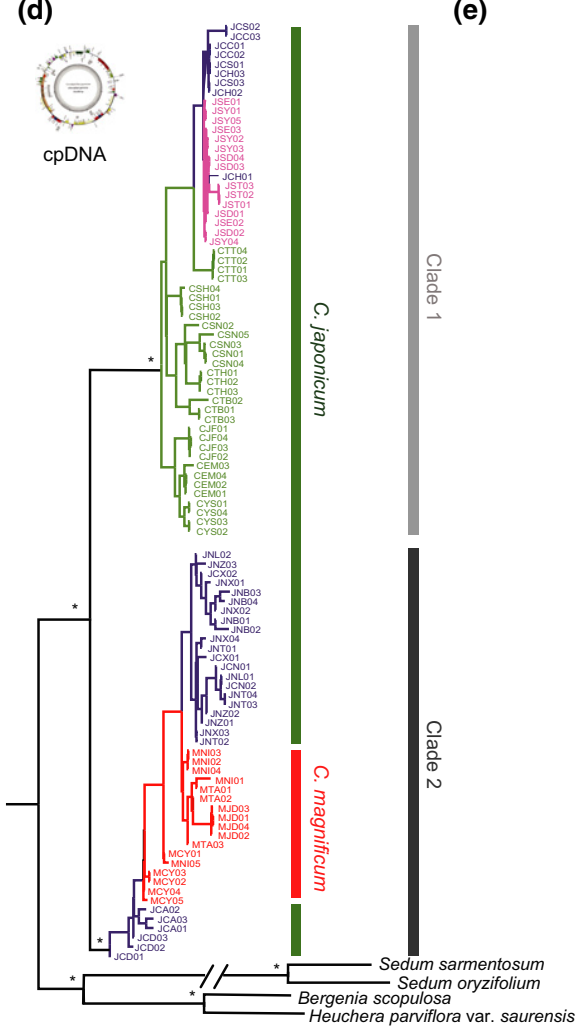

(b)
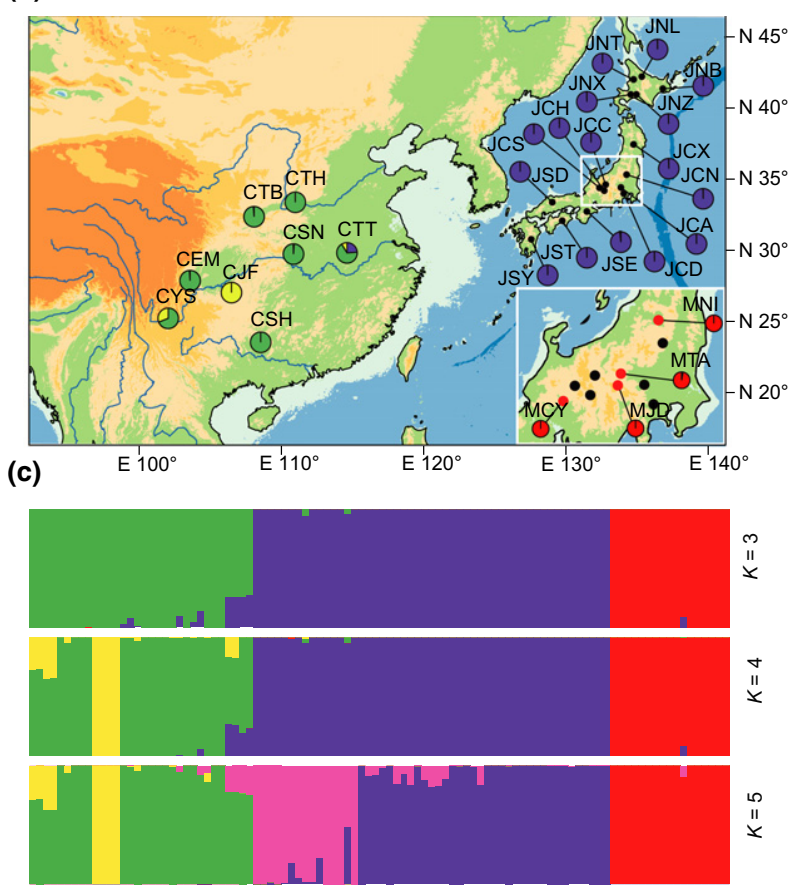

C. japonicum - China

C. japonicum - Japan

C. magnificum

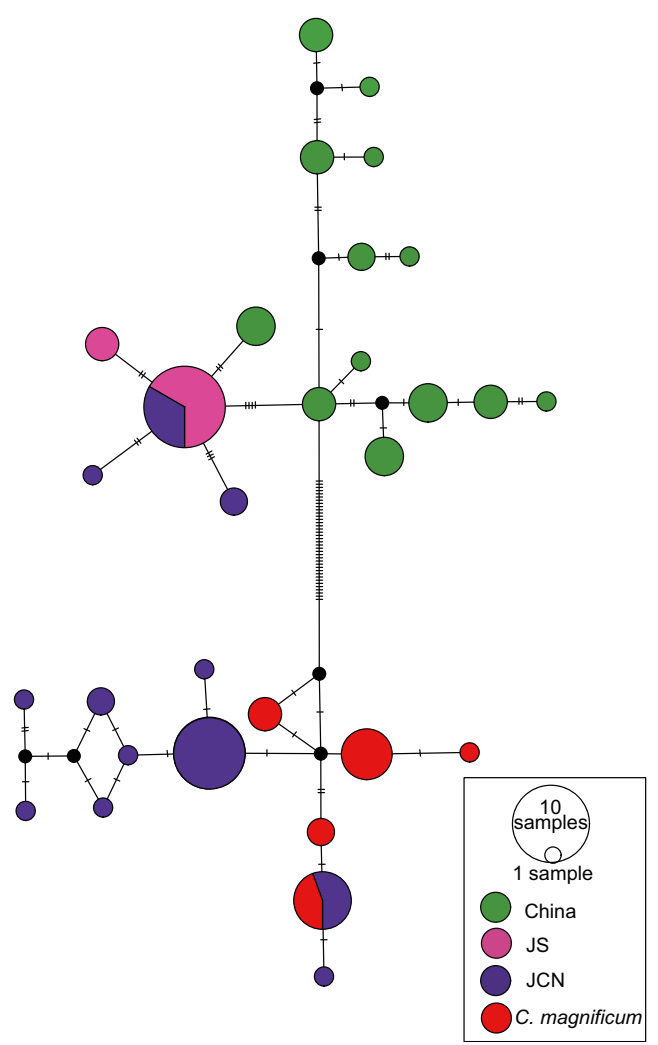

Fig. 3 Genetic relationships and population structure of Cercidiphyllum japonicum and Cercidiphyllum magnificum individuals based on whole-genome nuclear (nu) and chloroplast (cp) sequence data. (a) Phylogenetic relationships (maximumlikelihood tree) of all accessions $(n=99)$ inferred from 6923574 single nucleotide polymorphisms with $100 \%$ bootstrap support for almost all nodes. (b) Geographic distribution of ADMIXTURE clusters at $K=4$ (i.e. the optimal solution) within and among 24 populations of $C$. japonicum in China (eight) and Japan (16), and four populations of C. magnificum in Central Honshu/Japan (see inset). (c) Population structure of 99

Cercidiphyllum individuals based on ADMIXTURE analysis with $K=3,4$ and 5 . (d) Maximum likelihood phylogeny of all accessions ( $n=99$ ) based on whole-cp genome sequences. Asterisks indicate nodes with $>90 \%$ bootstrap support. (e) The $95 \%$ statistical parsimony (TCS) network of genealogical relationships between the $30 \mathrm{cp}$ haplotypes identified by considering coding sequence regions only. The sizes of circles are approximately proportional to sample sizes $n$, with the smallest circles representing $n=1$ and the largest representing $n=10$.
(Fig. 5c). This could indicate that Japanese vs Chinese populations were initially fixed for different alleles (type $I$ vs type $I$ ) due to strong selective sweeps and that a considerable number of Chinese individuals are recombinant hybrids due to introgression from Japanese C. japonicum.
Signatures of long-term balancing selection in C. japonicum

For C. japonicum, we identified regions putatively under balancing selection based on the $95 \%$ tail of Tajima's $D(D>1.3$ and 


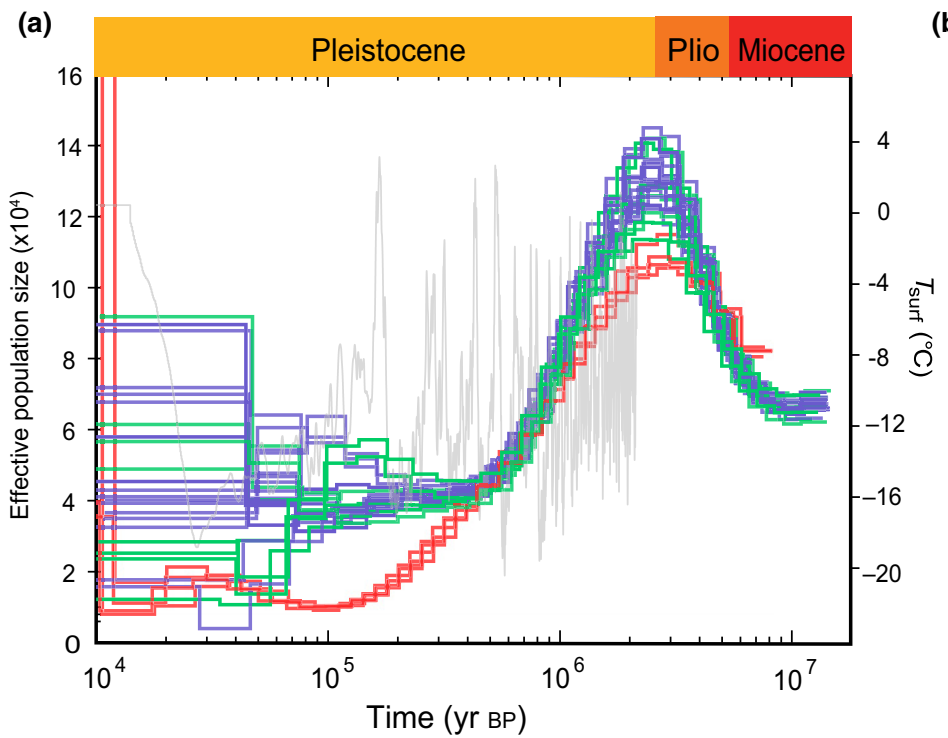

(b)

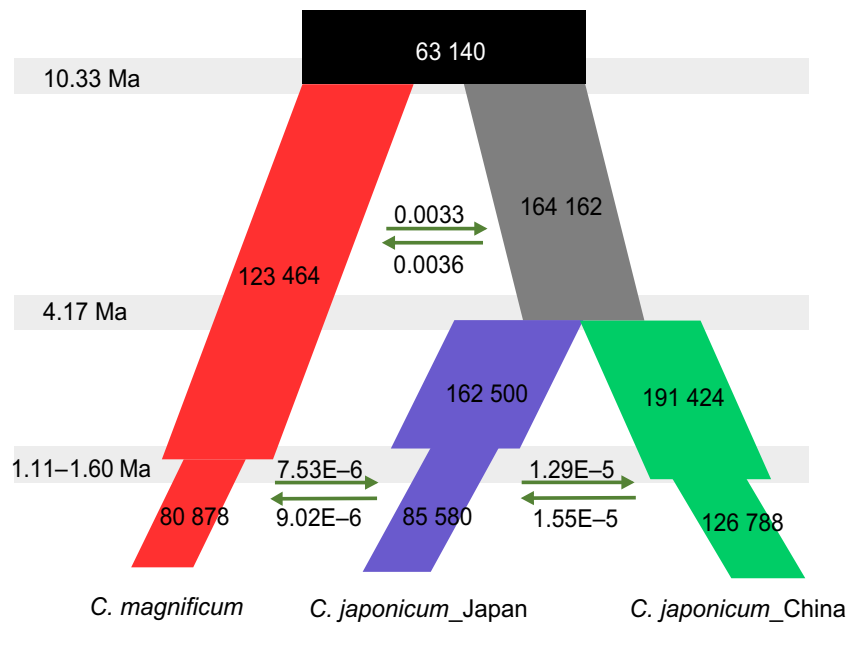

Fig. 4 Demographic history of the two Cercidiphyllum species. (a) Population size through time plot for 28 whole-genome resequenced individuals from each population of Cercidiphyllum japonicum (Japan: purple; China: green) and Cercidiphyllum magnificum (red) based on pairwise sequentially Markovian coalescent modeling. The atmospheric surface air temperature is given by the gray line. (b) Schematic of demographic scenarios modeled using FASTSIMCOAL2 with median split times in million years ago (Ma), estimates of effective population sizes $N_{\mathrm{e}}$ (in white within each segment), and migration rates (see Supporting Information Table S17 for 95\% confidence intervals). Estimates of gene flow between species and lineages are given as the migration fraction per generation. Chinese C. japonicum (green), Japanese C. japonicum (purple), their common ancestor (gray), and C. magnificum (red). A generation time $g$ of $10 \mathrm{yr}$ (Wan \& Zhang, 2002) and a mutation rate $\mu$ of $5.35 \times 10^{-9}$ per site per generation were applied (see Methods S2 for details).

$>1.56$ for Chinese and Japanese populations, respectively; SNPs $>100$ per $100 \mathrm{~kb}), \pi(>0.0024$ and $>0.0023$, respectively, $95 \%$ tail), and $F_{\mathrm{ST}}(z<-0.92,5 \%$ tail). Interestingly, most of these regions were shared by both Chinese and Japanese populations, especially for the four longest stretches on Chr03 (29.11-29.32 Mb), Chr16 (6.47-6.70 Mb), Chr17 (20.78-21.01 Mb), and Chr19 (21.31-21.79 Mb) (Table S19). Using normalized $\beta$ statistics, the presence of long-term balancing selection was confirmed in all four regions (Fig. 6b). We note that the mean read depths of all sites within those putative balancing selection regions (range: $19.78 \times-25.55 \times$ ) was similar to that of the whole genome $(21.08 \times$; see Fig. S15). Hence, the signatures of balancing selection detected herein cannot be a false signal due to exceptionally elevated read depths. Moreover, within these balancing selection regions, only a small percentage of sites (range: $0.8-5 \%$; see Fig. S16) were found to significantly deviate from Hardy-Weinberg equilibrium due to heterozygote excess (adjusted $P$-value $($ FDR $)<0.05$ ). After removing these sites, the percentages of heterozygous sites within these four regions increased to 35-51\% compared with $14 \%$ across the whole genome (Fig. 6a). Balanced polymorphisms in these four major regions were distributed in all phylogenetic clusters of Chinese and Japanese populations of C. japonicum, suggesting that balancing selection traced back to their common ancestor (Fig. 6c). Genes under balancing selection were related to the growth and development of flowers, pollen, leaves, and meristems (CLV2, PIN5) - for details on SNP genotypes and genes in these regions, see Fig. S17 and Table S20. No evidence of balancing selection was found in the same chromosomal regions of C. magnificum, suggesting that the balanced polymorphisms identified in C.japonicum are specific to this widespread Tertiary relict.

\section{Signatures of local adaptation in C. japonicum}

The PCA biplot of 19 bioclimatic variables completely separated Chinese and Japanese populations (Fig. S18; Table S21), whereby PC1 and PC2 (explaining $37.1 \%$ vs $32.1 \%$ of the total variance) were highly correlated with aspects of temperature (e.g. Bio1, Bio6, Bio11) and precipitation (e.g. Bio13, Bio16, Bio18), respectively (data not shown). Furthermore, the LFMM approach identified 5791 SNPs (representing 823 genes) showing significant genotype-climatic correlations based on FDR values $<0.05$ and LFMM scores $>10$. Of those $5791 \mathrm{SNPs}$, 1546 (representing 102 genes) were located in high $F_{\mathrm{ST}}$ ( $z$-score >2) chromosomal regions, and 1297 (83.9\%) were restricted to particular chromosomes (i.e. Chr02 (732 SNPs, seven genes), Chr14 (349 SNPs, eight genes), Chr07 (128 SNPs, eight genes), and Chr06 (88 SNPs, 10 genes)). Notably, on Chr14, the majority of SNPs (320/349; i.e. $91.7 \%)$ were located in the 'selective sweep' region at $8.5-12 \mathrm{Mb}$. These findings suggest that environmental differences could play a significant role in the selective sweep on Chr14 for different haplotypes in China and Japan. GO enrichment analyses of the 823 genes detected 109 significantly overrepresented GO terms $(\mathrm{FDR}<0.05$; Table S22). These GO categories were associated with reproduction, cellular development and proliferation, auxin metabolism and stress response (Fig. S19). Among those genes, a few were enriched for different functions, such as pollen formation (MGPI) and root hair elongation (VLN4) (on Chr14 and 
(a)

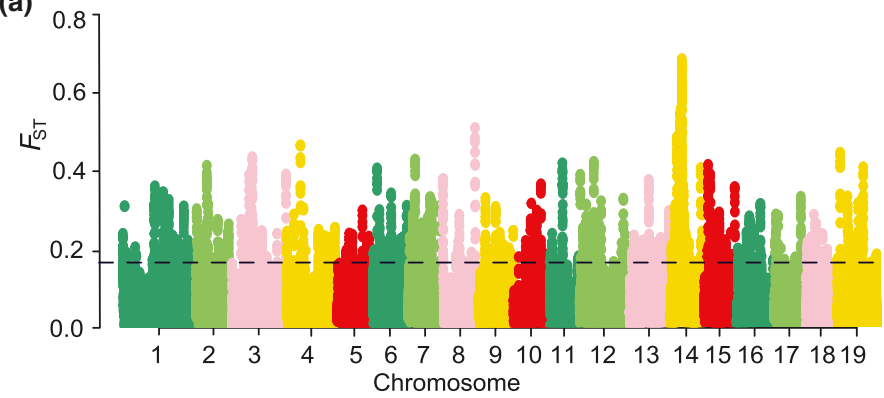

(c)

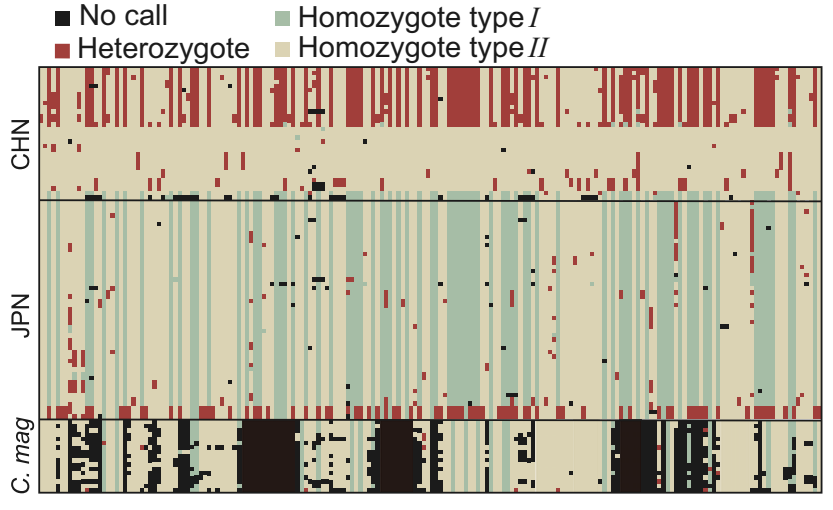

(b)

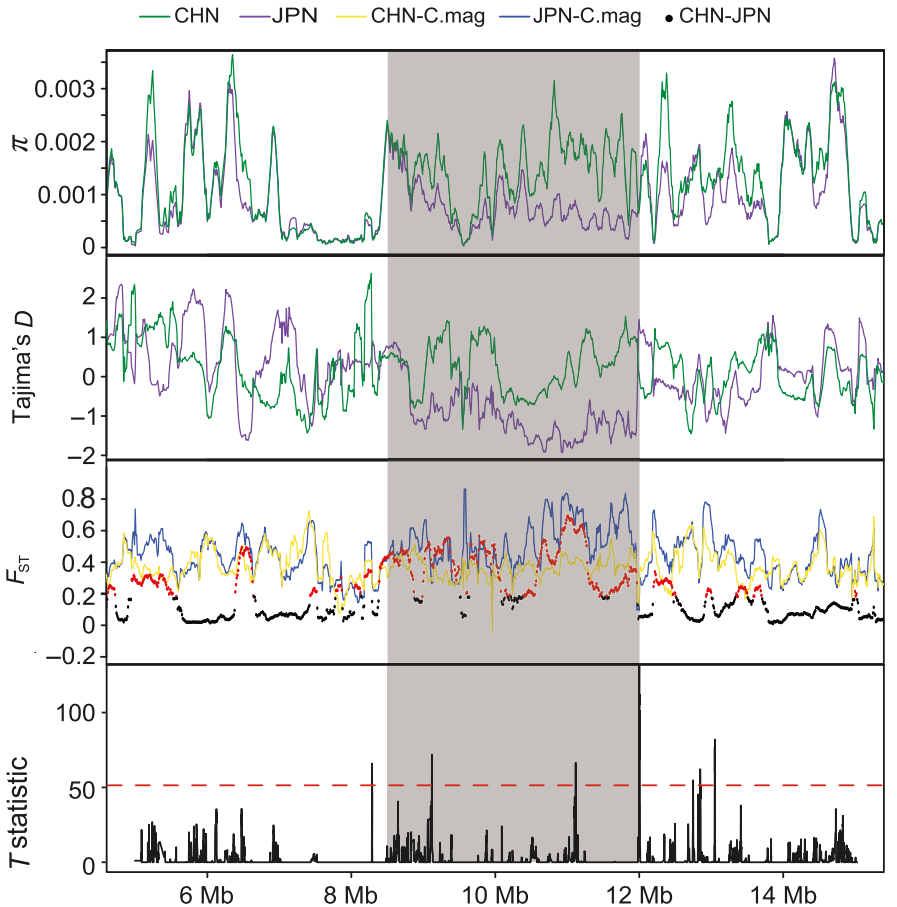

Fig. 5 Genomic differentiation and selection between Chinese and Japanese Cercidiphyllum japonicum. (a) $F_{\mathrm{ST}}$ values per chromosome (numbers 1-19) and chromosomal region between Chinese and Japanese $C$. japonicum. The dashed line marks the regions with $z$-score-normalized $F_{\mathrm{ST}}>2$. (b) Detection of putative selective sweep regions on chromosome (Chr)14 at 8.5-12 Mb (gray window). Estimates of nucleotide diversity $\pi$ and Tajima's $D$ for Chinese $(\mathrm{CHN})$ and Japanese (JPN) C. japonicum are represented by green and purple lines, respectively. Estimates of $F_{\mathrm{ST}}$ between Chinese and Japanese C. japonicum are represented by red $\left(z\right.$-score $\left.\left(F_{\mathrm{ST}}\right)>2\right)$ and black $\left(z\right.$-score $\left.\left(F_{\mathrm{ST}}\right)<2\right)$ dots, respectively, and those between either group and Cercidiphyllum magnificum by yellow and blue lines, respectively. Also shown are results of the likelihood ratio $T$ statistic (Harris \& DeGiorgio, 2019) for detecting putative selective sweep regions on Chr14 based on $T>51.9$ (99.5\% tail; red dashed line). (c) Single nucleotide polymorphism (SNP) genotypes of gene VLN4 (Chr14: 11.21-11.23 Mb) located within the putative selective sweep region of Chr14 (8.5-12 Mb; see Supporting Information Fig. S14) in Chinese (CHN) and Japanese (JPN) populations of C. japonicum and C. magnificum. The black, green, brown, and red grids indicate SNPs of no call, homozygote type $I$, homozygote type $I I$, and heterozygote, respectively.

Chr07, respectively), the regulation of photosynthesis (CGR3, $K E A 3)$, and vegetative phase change (CGR3, $S Q N-$ on Chr06), or possibly the circadian-clock pathway (PCL1, BOA - on Chr02; for details see Table S23).

\section{Discussion}

\section{Divergence and demographic history of Cercidiphyllum}

Our previous fossil-calibrated chloroplast DNA (cpDNA) phylogeny of 'woody Saxifragales' (Qi et al., 2012) suggested that the two extant species of Cercidiphyllum diverged at the Mio-Pliocene boundary, c. $6.53 \mathrm{Ma}$; however, this point estimate was afflicted with very large margins of error, amounting to many millions of years (95\% HPD: 13.42-1.34 Ma). By contrast, we here dated the divergence of C.japonicum and C. magnificum to around the mid-Miocene, whether based on the fossil-calibrated 287 singlecopy-gene phylogeny (c. $13.41 \mathrm{Ma}$ (95\% HPD: 15.0211.77 Ma); Fig. 2c) or population genomic (FASTSIMCOAL2) analyses (c. 10.33 Ma (95\% HPD: 10.96-6.73 Ma); Fig. 4b). The similarity of both latter estimates and their relatively narrow confidence limits suggest that the temporal scale of Cercidiphyllum speciation inferred herein (mid-Miocene) may be more accurate than the one (Mio-Pliocene boundary) based on cpDNA only (Qi et al., 2012). Our newly estimated divergence time coincides with the increasingly colder and arid climates following the midMiocene Climatic Optimum (MMCO, c. 17-15 Ma; Pound et al., 2012; Lin et al., 2016). Likewise, we here established with high confidence that the Chinese and Japanese lineages of C. japonicum split during the Early Pliocene, c. $4.17 \mathrm{Ma}$ (95\% HPD: 4.88-3.93 Ma; Fig. 4b); that is, shortly after the Japanese islands, for the first time, had completely separated from Eurasia c. $5 \mathrm{Ma}$ (Kimura, 2003). Our PSMC modeling (Fig. 4a) further indicated that each splitting event was initially accompanied by a drastic reduction in effective population size $N_{\mathrm{e}}$, resulting in increased genomic differentiation between the two Cercidiphyllum species and among the Chinese and Japanese lineages of C. japonicum (Qi et al., 2014; Cao et al., 2016). Taken together, these data support the notion that increasingly colder and arid climates following the MMCO not only caused contraction and fragmentation of numerous Tertiary relict trees in East Asia (Yabe et al., 2018), but also fostered their speciation and lineage diversification (e.g. Cercidiphyllum, this study; T. sinense: Sun et al., 2014; Cyclocarya paliurus: Kou et al., 2016; Juglans 
(a)

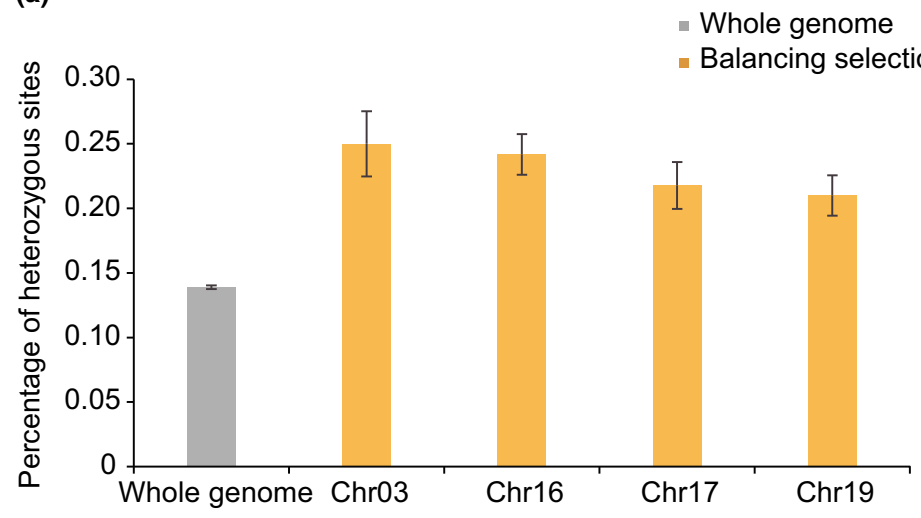

(c)

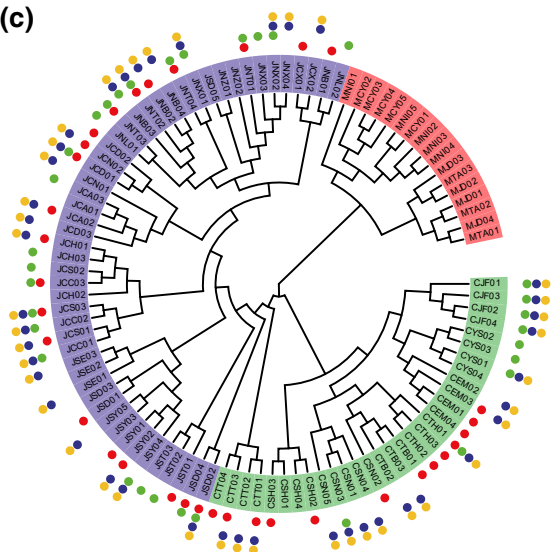

(b)

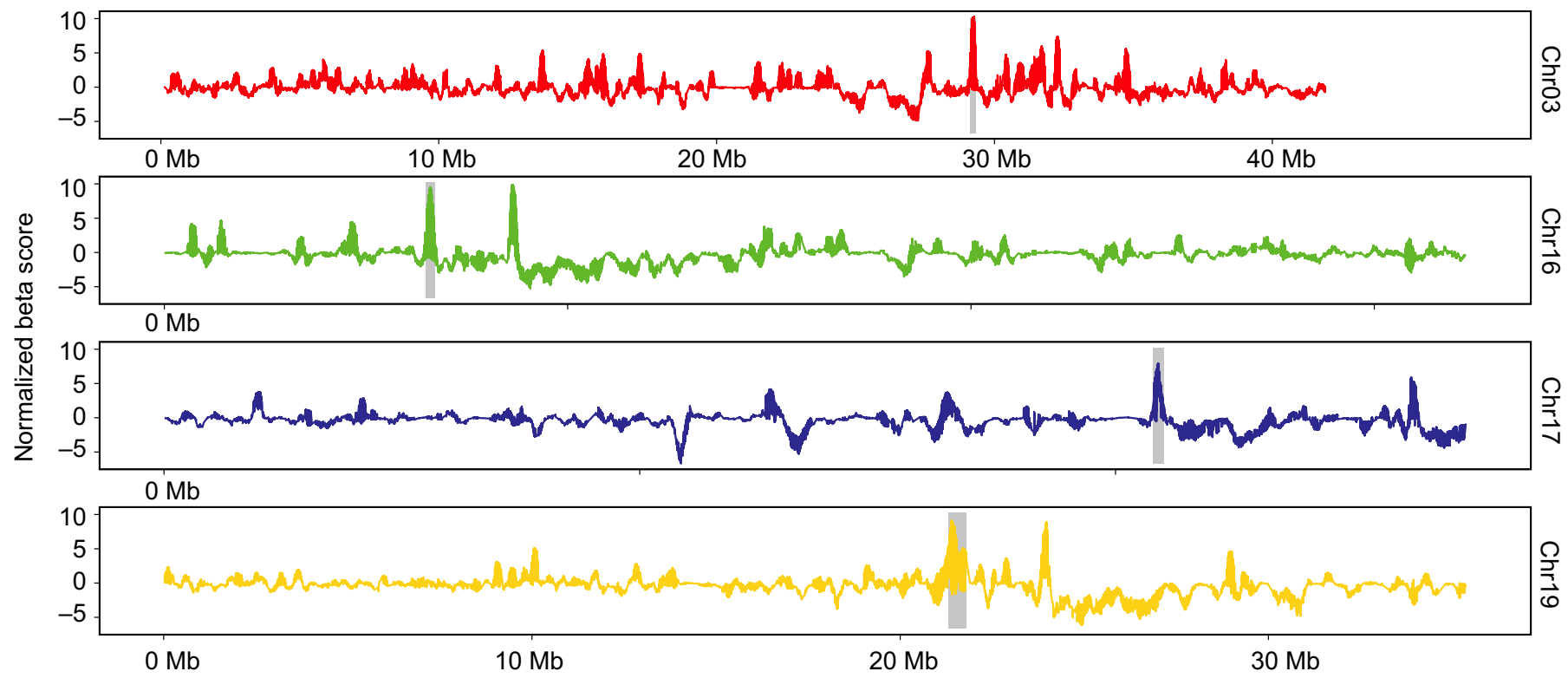

Fig. 6 Characterization of balancing selection in Cercidiphyllum japonicum. (a) Percentages of heterozygous sites in the whole genome and in the balancing selection regions on chromosome (Chr)03 (29.11-29.32 Mb), Chr16 (6.47-6.70 Mb), Chr17 (20.78-21.01 Mb), and Chr19 (21.31-21.79 Mb). The error bars indicate SE. (b) Balancing selection regions (gray windows) on Chr03 (29.11-29.32 Mb), Chr16 (6.47-6.70 Mb), Chr17 (20.78-21.01 Mb), and Chr19 (21.31-21.79 Mb) were also identified by normalized $\beta$ statistics. (c) Single nucleotide polymorphism-derived maximum-likelihood tree of Cercidiphyllum (the same as in Fig. 3a) highlighting C. japonicum heterozygous individuals putatively under balancing selection on different chromosomes, as indicated by colored dots (red: Chr03; green: Chr16; blue: Chr17; yellow: Chr19). Individuals were considered carriers of balancing polymorphism only if the percentage of heterozygous sites within regions was $>30 \%$.

spp.: Bai et al., 2018). However, when combined, our PSMC (Fig. 4a) and FASTSIMCOAL2 (Fig. 4b) analyses suggest an overall demographic scenario in which each Cercidiphyllum species first experienced long-term population growth from shortly after its origin (c. $10 \mathrm{Ma}$ ) to the Plio-Pleistocene boundary (c. 3-2 Ma), followed by recurrent declines in $N_{\mathrm{e}}$ (and thus genetic diversity) but also recoveries during the climatic upheavals of the late Tertiary-Quaternary. Hence, the population sizes of these two relict species have not continuously decreased, as might be expected for living fossil taxa (Sakai, 1971); rather, they increased over much of their evolutionary history before starting to fluctuate in response to more recent environmental change. Evidently, such demographic insights could not have been deduced from the fossil record alone, given its limitation in taxonomic resolution and spatial coverage (Mao \& Liu, 2012). Therefore, our results challenge the oft-stated view of relative demographic stasis in living fossils' (e.g. Lidgard \& Love, 2018). Moreover, in line with previous molecular phylogeographic data (Qi et al., 2012), they indicate that the late Tertiary-Quaternary range history of C. japonicum was highly dynamic. As further discussed later herein, positive results from selective sweeps should therefore be expected, especially in this widespread species, not least because adaptations (and thus sweeps) often occur in response to environmental change; that is, when populations colonize new habitats and thereby experience reductions in effective population size (e.g. González-Martínez et al., 2017; Stephan, 2019). 
Genomic differentiation and signatures of local adaptation in C. japonicum

Fossil records provide convincing evidence that drastic climatic fluctuations since the Late Pliocene in the Japanese Islands, which continued into the Middle Pleistocene, caused extensive extinction of the Japanese Tertiary forest flora while restricting survivors to subtropical China (e.g. G. biloba, D. involucrata, Metasequoia glyptostroboides) (Manchester et al., 2009; Yabe et al., 2018). By contrast, both fossil data (e.g. Crane \& Stockey, 1985; Onoe, 1989) and our population genomic data (Fig. 4a) indicate that C. japonicum survived in both subtropical China and the Japanese islands during the Pleistocene glacial-interglacial cycles. However, although Chinese and Japanese C. japonicum already diverged c. $4 \mathrm{Ma}$ (Fig. 4b), our population genomic (FASTSIMCOAL2) analyses of nuclear SNP data recovered only very low levels of post-divergence gene flow between these two lineages $\left(1.55 \times 10^{-5} / 1.29 \times 10^{-5}\right.$; Fig. $\left.4 \mathrm{~b}\right)$. Such gene exchange might have been facilitated by the glacially exposed East China Sea land bridge (c. 2.0-1.3 Ma and 0.2-0.015 Ma; Kimura, 2003).

When combined with previous ecological niche models (Qi et al., 2012), our coalescent-based demographic analyses using PSMC (Fig. 4a) suggest that both Chinese and Japanese C. japonicum experienced range contractions/expansions over the last glacial-interglacial cycles, implying that populations had to adapt to newly colonized habitats. In this study, our analysis revealed extensive signals of selective sweeps or positive selection across the genome of $C$. japonicum, particularly regarding signs of local adaptation to environmental differences between China and Japan at genes related to growth, development, and stress response. These loci under positive selection appear to be nonrandomly distributed along chromosomes. For example, a large region of Chr14 (8.5-12.0 Mb) was shown to have experienced selective sweeps and to harbor genes with polymorphisms that are strongly correlated with environmental factors. Selective sweeps tend to reduce genetic diversity in a single population. However, in structured populations, where selective sweeps may lead to the fixation of different alleles in different parts of the range, this will increase overall genomic variability (Coop et al., 2009). Lineagespecific selective sweeps usually lead to rapid coalescence within populations (Cruickshank \& Hahn, 2014) and result in an excess of fixed polymorphisms $\left(D_{\mathrm{f}}\right)$. This agrees with our observation that all 189 fixed nucleotide site differences between Chinese and Japanese populations of C. japonicum were located in this selective sweep region of Chr14 (Fig. 5b). Interestingly, this particular sweep region also contained a higher proportion of beneficial mutations (in terms of DFE parameter $p_{\mathrm{b}}$ ) and higher average strength of selection on positively selected mutations $\left(S_{\mathrm{b}}\right)$ in Japanese populations than in those from China. This could indicate that Japanese $C$. japonicum responded more strongly to selection than its mainland counterpart, possibly due to greater range fluctuations over the last glacial cycle(s), and especially while adapting to extreme environments outside the species' subtropical niche in the island's far north (i.e. northern Honshu/Hokkaido) after inter-/postglacial expansion from southern refugia (see also Qi et al., 2012).

\section{Balancing selection contributed to adaptation}

Though selection usually results in a reduction of diversity at selected loci and linked gene regions, balancing selection usually maintains diversity at such loci, whereby various advantageous variants can be maintained over long time periods, if not across species boundaries (e.g. major histocompatibility complex in vertebrates; self-incompatibility loci in plants; mating-type loci in fungi; reviewed in Fijarczyk \& Babik, 2015). Yet, when compared with positive selection, it is more difficult to detect genomic footprints of stable and pervasive balancing selection (DeGiorgio et al., 2014; Fijarczyk \& Babik, 2015), as older alleles had more time to recombine and may lead to narrow signatures around selected sites (Teixeira et al., 2015). In our case, however, low overall genetic diversity and an excess of rare alleles caused by severe bottlenecks, as likely characteristic of 'living fossils' (Avise et al., 1994), made it easier to detect loci under balancing selection (i.e. through signatures of high diversity and positive Tajima's $D$ ).

In C. japonicum, signatures of balancing selection are scattered across different chromosomes (on Chr03, Chr16, Chr17 and Chr19), with many regions characterized by multiple genes in heterozygote state; this pattern could potentially reflect overdominance (heterozygote fitness advantage; Delph \& Kelly, 2014). Notably, some of the genes potentially under balancing selection in C.japonicum, such as CLAVATA2 (CLV2), are highly pleiotropic and could therefore have a major effect on fitness. In A. thaliana, the CLV2 gene (along with several other receptors) regulates shoot/flower meristem and flower organ development (Kayes \& Clark, 1998; Jeong et al., 1999), and has also been found to be highly polymorphic, possibly due to balancing selection (Shepard \& Purugganan, 2003). In fact, balancing selection has been shown to be responsible for the maintenance of advantageous genetic diversity, notably heterozygote advantage, in a variety of discrete plant traits, such as self-incompatibility, nuclearcytoplasmic gynodioecy, flower color, heterostyly, and heterodichogamy (Delph \& Kelly, 2014). Populations of C.japonicum predominantly occur in heterogeneous (i.e. riparian) habitats (Kubo et al., 2010; Qi et al., 2012), which could have provided selective pressure strong enough to maintain polymorphisms over long periods of time within each population. Moreover, balancing selection acting on CLV2 might have increased the species' fitness under different circumstances; for instance, by enhancing or reducing the strength of signaling for different alleles involved in organ development (Shepard \& Purugganan, 2003; Turelli \& Barton, 2004). Our findings thus indicate that, in C. japonicum, genetic adaptation to, for example, disturbed or newly encountered environments may also involve ancestral polymorphisms, except for younger advantageous polymorphisms maintained by positive selection. Recent studies have demonstrated that balancing selection is playing crucial roles in adaptation and evolutionary processes driving genetic diversity and population differentiation (A. thaliana, Capsella rubella: Wu et al., 2017; Brachypodium distachyon: Bourgeois et al., 2018; Boechera stricta: Wang et al., 2019). Nevertheless, these studies have generally focused on model species, including very few forest trees 
(Populus. Wang et al., 2020). Our genomic study thus provides the first evidence for balancing selection acting on ancestral polymorphisms in a 'living fossil' tree.

\section{Conclusions}

Understanding how genetic diversity is maintained or lost through selective and neutral evolutionary forces has important implications for explaining current patterns of biodiversity and predicting future adaptive potential (e.g. Wang, 2010; Leffler et al., 2012). Until recently, however, this question has been largely restricted to model systems with abundant genomic resources, which may have biased our understanding of how the genomes of nonmodel species were shaped by evolutionary pressures (Bourgeois et al., 2018). Our population genomic analyses in Cercidiphyllum inferred a mid-Miocene speciation event, whereas the Chinese and Japanese lineages of C.japonicum diverged at the Early Pliocene. Although population bottlenecks during the climatic upheavals of the late Tertiary-Quaternary $(<3-2 \mathrm{Ma})$ greatly reduced the genetic diversity of $C$. japonicum, long-term balancing selection (most likely in the form of overdominance) and selective sweeps associated with local adaptation likely counteracted polymorphism loss. In fact, both types of selection could be major factors contributing to the species' long-term survival, resilience, and persistence in the face of past (and current) climate change. Overall, these results undercut the earlier assumption of relative demographic and evolutionary stasis in 'living fossils', in general (Sakai, 1971; Lidgard \& Love, 2018), and in an exceptionally widespread Tertiary relict tree in particular.

\section{Acknowledgements}

We thank Mingshui Zhao and Chunmei Pang (Tianmu Mountain National Nature Reserve) for assistance with sampling, LetPub (https://www.letpub.com) for linguistic assistance during the preparation of this manuscript, and Jianquan Liu (Sichuan University, China), Kenneth M. Olsen (University of Washington), and three reviewers for their constructive suggestions on previous versions of this manuscript. This work was supported by the National Key R\&D Program of China (2017YFA0605100) and the National Natural Science Foundation of China (31872652, 31561143015 and 31511140095).

\section{Author contributions}

YQ planned and designed the research. SZ, PL, RL, CF, WX, YF, WY, SS and YI participated in various aspects of biological sample collection. SZ performed experiments. JC, SZ, JZ and XX analyzed data. JC, SZ, HPC and YQ wrote the manuscript. LL, ML, SS and YI gave insightful suggestions and comments on the manuscript. SZ, JC and JZ contributed equally to this work.

\section{Data availability statement}

The assembled genome of C.japonicum and all raw sequencing data have been deposited under NCBI BioProject PRJNA549370 with accession nos. SRR 9640518-SRR 9640530. The transcriptomic raw data have been deposited under NCBI BioProject PRJNA554204 with accession nos. SAMN12240873SAMN12240879. The whole-genome resequencing raw data have been deposited under NCBI BioProject PRJN553317 with accession nos. SAMN12163473-SAMN12163572. The wholegenome SNP data sets and summary statistics have been deposited in GitHub (https://github.com/Cercidiphyllum/popu lation-genomics-analysis-of-Cercidiphyllum).

\section{ORCID}

Jun Chen (D) https://orcid.org/0000-0003-3187-7668

Hans Peter Comes (D) https://orcid.org/0000-0002-2659-8069

Yuji Isagi (D) https://orcid.org/0000-0002-9777-076X

Martin Lascoux (D) https://orcid.org/0000-0003-1699-9042

Linfeng Li (D) https://orcid.org/0000-0003-2820-3187

Pan Li (iD https://orcid.org/0000-0002-9407-7740

Yingxiong Qiu (iD https://orcid.org/0000-0002-4982-4997

Shota Sakaguchi (D) https://orcid.org/0000-0002-6180-1747

Xiao Xie (ID https://orcid.org/0000-0002-5241-6900

Jing Zhao (iD https://orcid.org/0000-0002-7449-124X

Shanshan Zhu (iD https://orcid.org/0000-0003-1582-1007

\section{References}

Alexander DH, Novembre J, Lange K. 2009. Fast model-based estimation of ancestry in unrelated individuals. Genome Research 19: 1655-1664.

Avise JC, Nelson WS, Sugita H. 1994. A speciational history of "living fossils": molecular evolutionary patterns in horseshoe crabs. Evolution 48: 1986-2001.

Axelrod DI. 1998. The Eocene Thunder Mountain flora of central Idaho. Berkeley, CA, USA: University of California Press.

Bai WN, Yan PC, Zhang BW, Woeste KE, Lin K, Zhang DY. 2018.

Demographically idiosyncratic responses to climate change and rapid Pleistocene diversification of the walnut genus Juglans (Juglandaceae) revealed by whole-genome sequences. New Phytologist 217: 1726-1736.

Bernatchez L. 2016. On the maintenance of genetic variation and adaptation to environmental change: considerations from population genomics in fishes. Journal of Fish Biology 89: 2519-2556.

Bourgeois Y, Stritt C, Walser JC, Gordon SP, Vogel JP, Roulin AC. 2018. Genome-wide scans of selection highlight the impact of biotic and abiotic constraints in natural populations of the model grass Brachiopodium distachyon. The Plant Journal 96: 438-451.

Cao YN, Comes HP, Sakaguchi S, Chen LY, Qiu YX. 2016. Evolution of East Asia's Arcto-Tertiary relict Euptelea (Eupteleaceae) shaped by Late Neogene vicariance and Quaternary climate change. BMC Evolutionary Biology 16: e66.

Castellano D, James J, Eyre-Walker A. 2018. Nearly neutral evolution across the Drosophila melanogaster genome. Molecular Biology and Evolution 35: 26852694.

Castresana J. 2000. Selection of conserved blocks from multiple alignments for their use in phylogenetic analysis. Molecular Biology and Evolution 17: 540-552.

Caye K, Jumentier B, Lepeule J, François O. 2019. LfmM 2: fast and accurate inference of gene-environment associations in genome-wide studies. Molecular Biology and Evolution 36: 852-860.

Coop G, Pickrell JK, Novembre J, Kudaravalli S, Li J, Absher D, Myers RM, Cavalli-Sforza LL, Feldman MW, Pritchard JK. 2009. The role of geography in human adaptation. PLoS Genetics 5: e1000500.

Crane PR, Stockey RA. 1985. Growth and reproductive biology of Joffrea speirsii gen. et sp. nov., a Cercidiphyllum-like plant from the Late Paleocene of Alberta, Canada. Canadian Journal of Botany 63: 340-364. 
Cruickshank TE, Hahn MW. 2014. Reanalysis suggests that genomic islands of speciation are due to reduced diversity, not reduced gene flow. Molecular Ecology 23: 3133-3157.

Dai X, Hu Q, Cai Q, Feng K, Ye N, Tuskan GA, Milne R, Chen Y, Wan Z, Wang $\mathrm{Z}$ et al. 2014. The willow genome and divergent evolution from poplar after the common genome duplication. Cell Research 24: 1274-1277.

Danecek P, Auton A, Abecasis G, Albers CA, Banks E, Depristo MA, Handsaker RE, Lunter G, Marth GT, Sherry ST et al. 2011. The variant call format and VCFTools. Bioinformatics 27: 2156-2158.

Darwin C. 1859. On the origin of species. Reprint 1996, Oxford, UK: Oxford University Press.

DeGiorgio M, Lohmueller KE, Nielsen R. 2014. A model-based approach for identifying signatures of ancient balancing selection in genetic data. PLoS Genetics 10: e1004561.

Delph LF, Kelly JK. 2014. On the importance of balancing selection in plants. New Phytologist 201: 45-56.

Drummond AJ, Suchard MA, Xie D, Rambaut A. 2012. Bayesian phylogenetics with BEAUTI and the BEAST 1.7. Molecular Biology and Evolution 29: 19691973.

Du Z, Zhou X, Ling Y, Zhang Z, Su Z. 2010. AGriGO: a GO analysis toolkit for the agricultural community. Nucleic Acids Research 38(Suppl. 2): W64-W70.

Edgar RC. 2004. MusCLE: multiple sequence alignment with high accuracy and high throughput. Nucleic Acids Research 32: 1792-1797.

Excoffier L, Dupanloup I, Huerta-Sánchez E, Sousa VC, Foll M. 2013. Robust demographic inference from genomic and SNP data. PLoS Genetics 9: e1003905.

Eyre-Walker A, Keightley PD. 2007. The distribution of fitness effects of new mutations. Nature Reviews Genetics 8: 610-618.

Fijarczyk A, Babik W. 2015. Detecting balancing selection in genomes: limits and prospects. Molecular Ecology 24: 3529-3545.

Fu D, Endress P. 2001. Cercidiphyllaceae. In: Wu ZY, Raven P, eds. Flora of China, vol. 6. Beijing, China/St Louis, MO, USA: Science Press/Missouri Botanical Science Press, 126.

González-Martínez SC, Ridout K, Pannell JR. 2017. Range expansion compromises adaptive evolution in an outcrossing plant. Current Biology 27: 2544-2551.

Gossmann TI, Shanmugasundram A, Börno S, Duvaux L, Lemaire C, Kuhl H, Klages S, Roberts LD, Schade S, Gostner JM et al. 2019. Ice-age climate adaptations trap the alpine marmot in a state of low genetic diversity. Current Biology 29: 1712-1720.

Grandcolas P, Nattier R, Trewick S. 2014. Relict species: a relict concept? Trends in Ecology and Evolution 29: 655-663.

Han MV, Thomas GW, Lugo-Martinez J, Hahn MW. 2013. Estimating gene gain and loss rates in the presence of error in genome assembly and annotation using CAFE 3. Molecular Biology and Evolution 30: 1987-1997.

Harris AM, DeGiorgio M. 2019. A likelihood approach for uncovering selective sweep signatures from haplotype data. bioRxiv: 678722.

Hedrick PW. 2006. Genetic polymorphism in heterogeneous environments: the age of genomics. Annual Review of Ecology, Evolution, and Systematics 37: 67-93.

Hijmans R, Cameron S, Parra J, Jones P, Jarvis A, Richardson K. 2005. Very high resolution interpolated climate surfaces for global land areas. International Journal of Climatology: A Journal of the Royal Meteorological Society 15: 1965-1978.

Huang DI, Hefer CA, Kolosova N, Douglas CJ, Cronk QC. 2014. Whole plastome sequencing reveals deep plastid divergence and cytonuclear discordance between closely related balsam poplars, Populus balsamifera and $P$. trichocarpa (Salicaceae). New Phytologist 204: 693-703.

Isagi Y, Kudo M, Osumi K, Sato T, Sakio H. 2005. Polymorphic microsatellite DNA markers for a relictual angiosperm Cercidiphyllum japonicum Sieb. et Zucc. and their utility for Cercidiphyllum magnificum. Molecular Ecology Notes 5: 596-598.

Jaillon O, Aury J-M, Noel B, Policriti A, Clepet C, Casagrande A, Choisne N, Aubourg S, Vitulo N, Jubin C et al. 2007. The grapevine genome sequence suggests ancestral hexaploidization in major angiosperm phyla. Nature 449: 463-467.

James J, Castellano D, Eyre-Walker A. 2017. DNA sequence diversity and the efficiency of natural selection in animal mitochondrial DNA. Heredity 118: $88-95$.
Jeong S, Trotochaud AE, Clark SE. 1999. The Arabidopsis CLAVATA2 gene encodes a receptor-like protein required for the stability of the CLAVATA1 receptor-like kinase. The Plant Cell 11: 1925-1934.

Kayes JM, Clark SE. 1998. CLAVATA2, a regulator of meristem and organ development in Arabidopsis. Development 119: 3843-3851.

Kern AD, Hahn MW. 2018. The neutral theory in light of natural selection. Molecular Biology and Evolution 35: 1366-1371.

Kimura M. 2003. Land connections between Eurasian continent and Japanese islands - related to human migration. Migration and Diffusion 4: 14-33.

Kou Y, Cheng S, Tian S, Li B, Fan D, Chen Y, Soltis DE, Soltis PS, Zhang Z. 2016. The antiquity of Cyclocarya paliurus (Juglandaceae) provides new insights into the evolution of relict plants in subtropical China since the late Early Miocene. Journal of Biogeography 43: 351-360.

Krassilov V. 2010. Cercidiphyllum and fossil allies: morphological interpretation and general problems of plant evolution and development. Moscow, Russia: Pensoft Press.

Kryvokhyzha D, Salcedo A, Eriksson MC, Duan T, Tawari N, Chen J, Guerrina M, Kreiner JM, Kent TV, Lagercrantz U et al. 2019. Parental legacy,

demography, and admixture influenced the evolution of the two subgenomes of the tetraploid Capsella bursa-pastoris (Brassicaceae). PLoS Genetics 15: e1007949.

Kubo M, Shimano K, Sakio H, Isagi Y, Ohno K. 2010. Difference between sprouting traits of Cercidiphyllum japonicum and C. magnificum. Journal of Forest Research 15: 337-340.

Leffler EM, Bullaughey K, Matute DR, Meyer WK, Ségurel L, Venkat A, Andolfatto P, Przeworski M. 2012. Revisiting an old riddle: what determines genetic diversity levels within species? PLoS Biology 10: e1001388.

Leigh JW, Bryant D. 2015. PopART: full-feature software for haplotype network construction. Methods in Ecology and Evolution 6: 1110-1116.

Li H, Durbin R. 2009. Fast and accurate short read alignment with BurrowsWheeler transform. Bioinformatics 25: 1754-1760.

Li H, Durbin R. 2010. Fast and accurate long-read alignment with BurrowsWheeler transform. Bioinformatics 26: 589-595.

Li H, Durbin R. 2011. Inference of human population history from individual whole-genome sequences. Nature 475: 493-496.

Li L, Stoeckert CJ, Roos DS. 2003. ORTHOMCL: identification of ortholog groups for eukaryotic genomes. Genome Research 13: 2178-2189.

Li Y, Willer CJ, Ding J, Scheet P, Abecasis GR. 2010. MACH: using sequence and genotype data to estimate haplotypes and unobserved genotypes. Genetic Epidemiology 34: 816-834.

Lidgard S, Love AC. 2018. Rethinking living fossils. BioScience 68: 760-770.

Lin X, Wyrwoll KH, Chen H, Cheng X. 2016. On the timing and forcing mechanism of a mid-Miocene arid climate transition at the NE margins of the Tibetan Plateau: stratigraphic and sedimentologic evidence from the Sikouzi Section. International Journal of Earth Sciences 105: 1039-1049.

Lin Y, Chen Y, Yi C, Fong JJ, Kim W, Rius M, Zhan A. 2017. Genetic signatures of natural selection in a model invasive ascidian. Scientific Reports 7: e44080.

Ma Q, Du YJ, Chen N, Zhang LY, Li JH, Fu CX. 2015. Phylogeography of Davidia involucrata (Davidiaceae) inferred from cpDNA haplotypes and nSSR data. Systematic Botany 40: 796-810.

Maere S, De Bodt S, Raes J, Casneuf T, Van Montagu M, Kuiper M, Van de Peer Y. 2005. Modeling gene and genome duplications in eukaryotes. Proceedings of the National Academy of Sciences, USA 102: 5454-5459.

Manchester SR, Chen ZD, Lu AM, Uemura K. 2009. Eastern Asian endemic seed plant genera and their paleogeographic history throughout the Northern Hemisphere. Journal of Systematics and Evolution 47: 1-42.

Mao KS, Liu JQ. 2012. Current 'relicts' more dynamic in history than previously thought. New Phytologist 196: 329-331.

Onoe T. 1989. Palaeoenvironmental analysis based on the Pleistocene Shiobara flora in the Shiobara volcanic basin, Central Japan. Reports of the Geological Survey of Japan 269: 1-207.

Parra G, Bradnam K, Korf I. 2007. CEGMA: a pipeline to accurately annotate core genes in eukaryotic genomes. Bioinformatics 23: 1061-1067.

Petit RJ, Aguinagalde I, de Beaulieu JL, Bittkau C, Brewer S, Chedaddi R, Ennos R, Fineschi S, Grivet D, Lascoux M et al. 2003. Glacial refugia: hotspots but not melting pots of genetic diversity. Science 300: 1563-1565. 
Posada D, Buckley TR. 2004. Model selection and model averaging in phylogenetics: advantages of Akaike information criterion and Bayesian approaches over likelihood ratio tests. Systematic Biology 53: 793-808.

Pound MJ, Haywood AM, Salzmann U, Riding JB. 2012. Global vegetation dynamics and latitudinal temperature gradients during the Mid to Late Miocene (15.97-5.33 Ma). Earth-Science Reviews 112: 1-22.

Price MN, Dehal PS, Arkin AP. 2010. FASTTREE 2 - approximately maximumlikelihood trees for large alignments. PLoS ONE 5: e9490.

Qi XS, Chen C, Comes HP, Sakaguchi S, Liu YH, Tanaka N, Sakio H, Qiu YX. 2012. Molecular data and ecological niche modelling reveal a highly dynamic evolutionary history of the East Asian Tertiary relict Cercidiphyllum (Cercidiphyllaceae). New Phytologist 196: 617-630.

Qi XS, Yuan N, Comes HP, Sakaguchi S, Qiu YX. 2014. A strong 'filter' effect of the East China Sea land bridge for East Asia's temperate plant species: inferences from molecular phylogeography and ecological niche modelling of Platycrater arguta (Hydrangeaceae). BMC Evolutionary Biology 14: e41.

Qiu YX, Fu CX, Comes HP. 2011. Plant molecular phylogeography in China and adjacent regions: tracing the genetic imprints of Quaternary climate and environmental change in the world's most diverse temperate flora. Molecular Phylogenetics and Evolution 59: 225-244.

Quick C, Fuchsberger C, Taliun D, Abecasis G, Boehnke M, Kang HM. 2018. EMERALD: rapid linkage disequilibrium estimation with massive datasets. Bioinformatics 35: 164-166.

R Development Core Team. 2018. $R$ : a language and environment for statistical computing. Vienna, Austria: R Foundation for Statistical Computing.

Sakai A. 1971. Freezing resistance of relicts from the Arcto-Tertiary flora. New Phytologist 70: 1199-1205.

Shang KK, Song K, Da LJ. 2016. Ecology of relict Tertiary deciduous trees in subtropical China. In: Box EO, ed. Vegetation structure and function at multiple spatial, temporal and conceptual scales. Cham, Switzerland: Springer, 149-167.

Shaw RG, Etterson JR. 2012. Rapid climate change and the rate of adaptation: insight from experimental quantitative genetics. New Phytologist 195: 752-765.

Shen Y, Cheng Y, Li K, Li H. 2019. Integrating phylogeographic analysis and geospatial methods to infer historical dispersal routes and glacial refugia of Liriodendron chinense. Forests 10: e565.

Shepard KA, Purugganan MD. 2003. Molecular population genetics of the Arabidopsis CLAVATA2 region: the genomic scale of variation and selection in a selfing species. Genetics 163: 1083-1095.

Siewert KM, Voight BF. 2017. Detecting long-term balancing selection using allele frequency correlation. Molecular Biology and Evolution 34: 2996-3005.

Simão FA, Waterhouse RM, Ioannidis P, Kriventseva EV, Zdobnov EM. 2015. BUSCO: assessing genome assembly and annotation completeness with singlecopy orthologs. Bioinformatics 31: 3210-3212.

Stamatakis A. 2014. RAxML version 8: a tool for phylogenetic analysis and postanalysis of large phylogenies. Bioinformatics 30: 1312-1313.

Stephan W. 2019. Selective sweeps. Genetics 211: 5-13.

Sun Y, Moore MJ, Yue L, Feng T, Chu H, Chen S, Ji Y, Wang H, Li J. 2014. Chloroplast phylogeography of the East Asian Arcto-Tertiary relict Tetracentron sinense (Trochodendraceae). Journal of Biogeography 41: 1721-1732.

Tataru P, Bataillon T. 2018. POLYDFEv2.0: testing for invariance of the distribution of fitness effects within and across species. bioRxiv: 363887.

Teixeira JC, de Filiopo C, Weihmann A, Meneu JR, Racimo F, Dannemann M, Nickel B, Fischer A, Halbwax M, Andre C et al. 2015. Long-term balancing selection in $L A D 1$ maintains a missense trans-species polymorphism in humans, chimpanzees, and bonobos. Molecular Biology and Evolution 325: 1186-1196.

Terhorst J, Kamm JA, Song YS. 2017. Robust and scalable inference of population history from hundreds of unphased whole genomes. Nature Genetics 49: 303-309.

Tsumura Y, Uchiyama K, Moriguchi Y, Ueno S, Ihara-Ujino T. 2012. Genome scanning for detecting adaptive genes along environmental gradients in the Japanese conifer, Cryptomeria japonica. Heredity 109: 349-360.

Turelli M, Barton N. 2004. Polygenic variation maintained by balancing selection: pleiotropy, sex-dependent allelic effects and $\mathrm{G} \times \mathrm{E}$ interactions. Genetics 166: 1053-1079.

Tuskan GA, Difazio S, Jansson S, Bohlmann J, Grigoriev I, Hellsten U, Putnam N, Ralph S, Rombauts S, Salamov A. 2006. The genome of black cottonwood, Populus trichocarpa (Torr. \& Gray). Science 313: 1596-1604.
Wan T, Zhang J. 2002. The growth law of the individual from artificial community of 10-year-old Cercidiphyllum japonicum. Journal of Sichuan Forestry Science and Technology 23: 34-38.

Wang BS, Mojica JP, Perera N, Li CR, Lovell JT, Sharma A, Adam C, Lipzen A, Barry K, Rokhsar DS et al. 2019. Ancient polymorphisms contribute to genome-wide variation by long-term balancing selection and divergent sorting in Boechera stricta. Genome Biology 20: e126.

Wang IJ. 2010. Recognizing the temporal distinctions between landscape genetics and phylogeography. Molecular Ecology 19: 2605-2608.

Wang K, Li M, Hakonarson H. 2010. ANNOVAR: functional annotation of genetic variants from high-throughput sequencing data. Nucleic Acids Research 38: e164-e164.

Wang MC, Zhang L, Zhang ZY, Li MM, Wang D, Zhang X, Xi ZX, KeefoverRing K, Smart LB, DiFazio SP et al. 2020. Phylogenomics of the genus Populus reveals extensive interspecific gene flow and balancing selection. New Phytologist 225: 1370-1382.

Wang Y, Tang H, DeBarry JD, Tan X, Li J, Wang X, Lee TH, Jin H, Marler B, Guo H. 2012. MCSCANX: a toolkit for detection and evolutionary analysis of gene synteny and collinearity. Nucleic Acids Research 40: e49.

Werth AJ, Shear WA. 2014. The evolutionary truth about living fossils. American Scientist 102: 434-443.

Wu Q, Han TS, Chen X, Chen JF, Zou YP, Li ZW, Xu YC, Guo YL. 2017. Long-term balancing selection contributes to adaptation in Arabidopsis and its relatives. Genome Biology 18: 217.

Yabe A, Jeong E, Kim K, Uemura K. 2018. Oligocene-Neogene fossil history of Asian endemic conifer genera in Japan and Korea. Journal of Systematics and Evolution 57: 114-128.

Zhang YH, Wang IJ, Comes HP, Peng H, Qiu YX. 2016. Contributions of historical and contemporary geographic and environmental factors to phylogeographic structure in a Tertiary relict species, Emmenopterys henryi (Rubiaceae). Scientific Reports 6: 24041.

Zhao YP, Fan G, Yin PP, Sun S, Li N, Hong X, Hu G, Zhang H, Zhang FM, Han JD et al. 2019. Resequencing 545 Ginkgo genomes across the world reveals the evolutionary history of the living fossil. Nature Communications 10 : e4201.

\section{Supporting Information}

Additional Supporting Information may be found online in the Supporting Information section at the end of the article.

Fig. S1 Gene family classification for 18 species (including C. japonicum) in comparative genomic analysis.

Fig. S2 Geographic distribution of sampling locations for C. japonicum in China and Japan $(n=24)$ and C. magnificum in Central Honshu/Japan $(n=4)$.

Fig. S3 Schematic diagrams of six alternative divergence models with different gene flow events among the three lineages of Cercidiphyllum (C. magnificum, Japanese C.japonicum, Chinese C. japonicum, their common ancestor) using FASTSIMCOAL2.

Fig. $\mathbf{S 4} K$-mer analysis for $C$. japonicum genome size estimation.

Fig. S5 Chromosomal Hi-C contact map of the C.japonicum genome.

Fig. S6 Syntenic depth patterns between C.japonicum vs Vitis vinifera and between C.japonicum vs Kalanchoe fedtschenkoi, respectively. 
Fig. S7 The expansion and contraction of gene families in C. japonicum and 17 other species.

Fig. S8 Genetic relationships (ML tree) and population structure of Cercidiphyllum japonicum and C. magnificum individuals based on whole-genome nuclear dataset ( $n=99 ; 2794617$ SNPs).

Fig. S9 Changes of effective population size $\left(N_{\mathrm{e}}\right)$ through time in C. japonicum estimated on its reference genome by using pairwise sequentially Markovian coalescent (PSMC) modelling.

Fig. S10 Changes in effective population size $\left(N_{\mathrm{e}}\right)$ through time in C. japonicum from China and Japan and C. magnificum using the SMC++ method.

Fig. S11 Schematic diagram of the best-fitting divergence model (no. 6) of Cercidiphyllum (C. magnificum, Japanese C. japonicum, Chinese C.japonicum, their common ancestor) as inferred by FASTSIMCOAL2.

Fig. S12 Genetic parameters for Cercidiphyllum species at the whole genome level.

Fig. S13 The linkage disequilibrium (LD) decay of C. japonicum all individuals, Chinese and Japanese C. japonicum individuals, respectively.

Fig. S14 SNP genotype variation of C.japonicum individuals from, respectively, China and Japan within the selective sweep region of chromosome 14 (Chr14) from $8.5 \mathrm{Mb}$ to $12 \mathrm{Mb}$, as depicted for different length intervals.

Fig. S15 Frequency distribution of reads depth within balancing selection regions of Chr03 (29.11-29.32 Mb), Chr16 (6.476.70 Mb), Chr17 (20.78-21.01 Mb) and Chr19 (21.31$21.79 \mathrm{Mb})$.

Fig. S16 Hardy-Weinberg Equilibrium (HWE) test for all sites in the balancing selection regions of Chr03 (29.11-29.32 Mb), Chr16 (6.47-6.70 Mb), Chr17 (20.78-21.01 Mb) and Chr19 (21.31-21.79 Mb).

Fig. S17 SNP genotype variation of C. japonicum individuals from, respectively, China and Japan within the balancing selection regions of $\mathrm{Chr} 03$ (29.11-29.32 Mb), Chr16 (6.476.70 Mb), Chr17 (20.78-21.01 Mb), and Chr19 (21.31$21.79 \mathrm{Mb})$.

Fig. S18 Principal component analysis (PCA) based on 19 bioclimatic variables of altogether 24 populations of $C$. japonicum from China $(n=8)$ and Japan $(n=16)$.

Fig. S19 The Gene Ontology (GO) terms with enrichment ratio more than 1 for genes significantly correlated with environmental variables in the genome of $C$. japonicum.
Methods S1 Genome assembly and genome evolution of Cercidiphyllum japonicum.

Methods S2 Population genomics analysis.

Table S1 Whole-genome sequencing data statistics of C. japonicum.

Table S2 Genome sizes estimated for C. japonicum based on 17mer statistics.

Table S3 Characteristics of DNA base composition within the genome of C. japonicum.

Table S4 Validation of the completeness of the C.japonicum genome, using the Core Eukaryotic Genes Mapping Approach (CEGMA) based on 248 ultra-conserved core eukaryotic genes (CEGs).

Table S5 Validation of the completeness of the C.japonicum genome, using the Benchmarking Universal Single Copy Orthologs (BUSCO) approach.

Table S6 Validation of the genome assembly of C.japonicum using reads mapping and transcript alignment, respectively.

Table S7 Statistics for RNA-seq data from five tissues of C. japonicum.

Table S8 Prediction of protein-coding gene models in the genome of C. japonicum.

Table S9 Functional annotation of the predicted genes within the genome of C. japonicum.

Table S10 Classification of transposable elements (TEs) identified in the C. japonicum genome, including their length (in bp), the percent of total repeats and the percent of genome.

Table S11 Genomic comparison of gene families across 18 plant species, including C. japonicum.

Table S12 Gene ontology (GO) enrichment analysis of the expanded gene families in C. japonicum.

Table S13 PFAM functional enrichment analysis of the expanded gene families in C. japonicum.

Table S14 Sample information and sequencing statistics for population genomics of C. japonicum and C. magnificum.

Table S15 Annotation of single nucleotide polymorphism (SNP) variants across the 99 re-sequenced individuals of Cercidiphyllum, using ANNOVAR (Wang et al., 2010) and with different filtering criteria. 
Table S16 Comparison of six divergence models analysed with FASTSIMCOAL2.

Table S17 Parameter estimates with 95\% highest posterior density (HPD) intervals for the best divergence model (no. 6) with parameter tags corresponding to Fig. S11.

Table S18 The 41 protein coding genes located in a region stretching from $5 \mathrm{Mb}$ to $14 \mathrm{Mb}$ of Chromosome 14 with statistics of nonsynonymous and synonymous sites in Chinese and Japanese C. japonicum.

Table S19 Putative regions under balancing selection in Chinese and Japanese populations of C. japonicum.

Table S20 Genes under balancing selection shared by both Chinese and Japanese populations of C. japonicum.
Table S21 Nineteen bioclimatic variables downloaded from WORLDCLIM.

Table S22 Gene Ontology (GO) enrichment analysis of the genes associated with environmental factors in C.japonicum using LFMM.

Table S23 The genes are correlated with environmental factors and located within high $F_{S T} \quad(z \quad$ score $>2)$ regions in C. japonicum.

Please note: Wiley Blackwell are not responsible for the content or functionality of any Supporting Information supplied by the authors. Any queries (other than missing material) should be directed to the New Phytologist Central Office. 